Supporting Information; Appendix 1: Nilsson and Overman Supporting Information: Bradley L. Nilsson and Larry E. Overman Appendix 1: ${ }^{1}$ Hand ${ }^{13}$ C NMR Spectra, Part I

\title{
Concise Synthesis of Guanidine-Containing Heterocycles Using the Biginelli Reaction
}

\author{
Bradley L. Nilsson and Larry E. Overman*
}

Department of Chemistry, 516 Rowland Hall, University of California, Irvine, CA 92697-2025

Spectra for compounds 18a-f, 19a-f, 16a-f, 10a-f, 10i, 25, 26, 28, 20, 21a-j 
Supporting Information; Appendix 1: Nilsson and Overman 6-Methyl-4-phenyl-2-pyrazol-1-yl-1,4-dihydropyrimidine-5-carboxylic acid ethyl ester (Compound 18a).

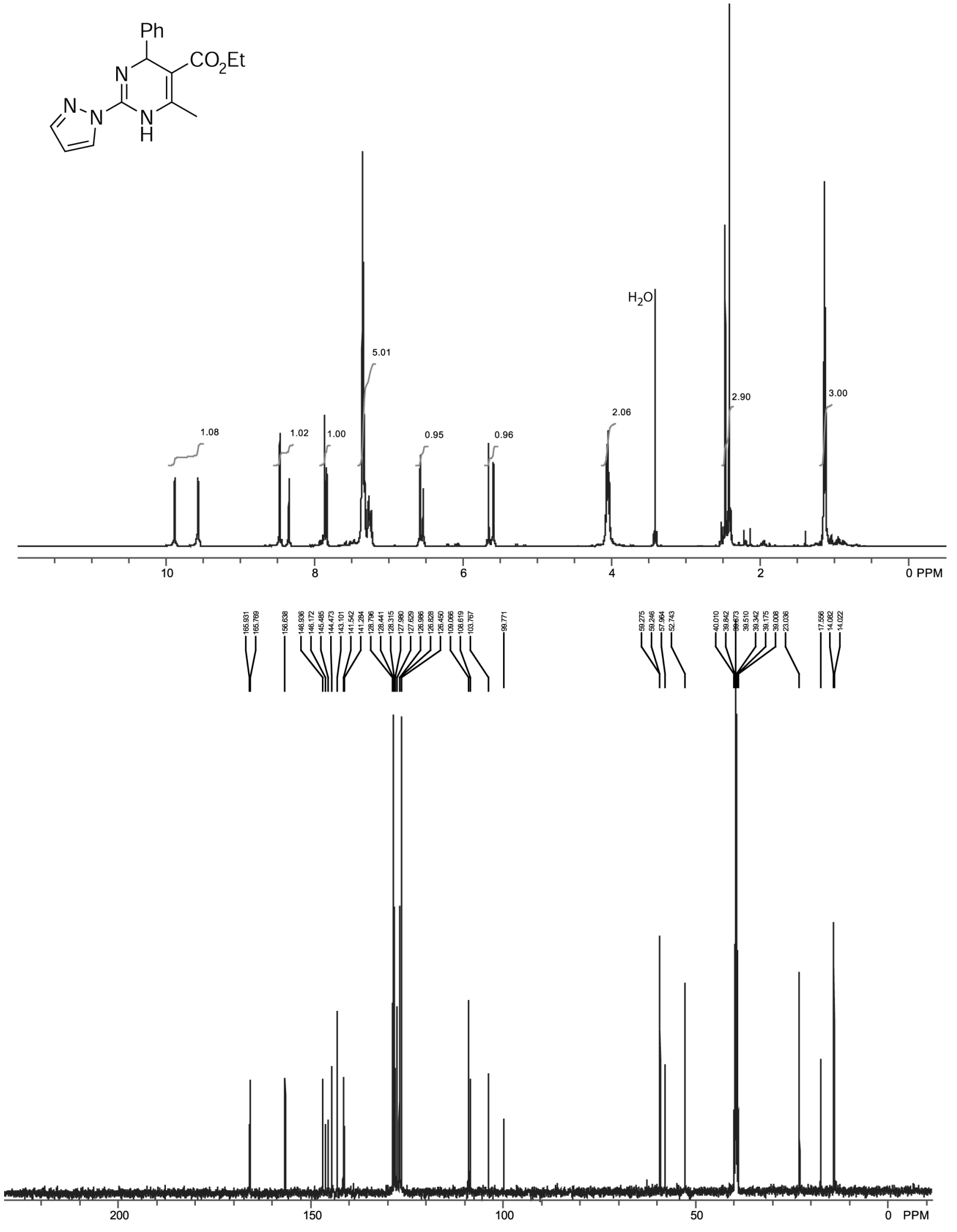



6-Methyl-2-pyrazol-1-yl-4-(2-vinylphenyl)-1,4-dihydropyrimidine-5-carboxylic acid ethyl ester (Compound 18b).
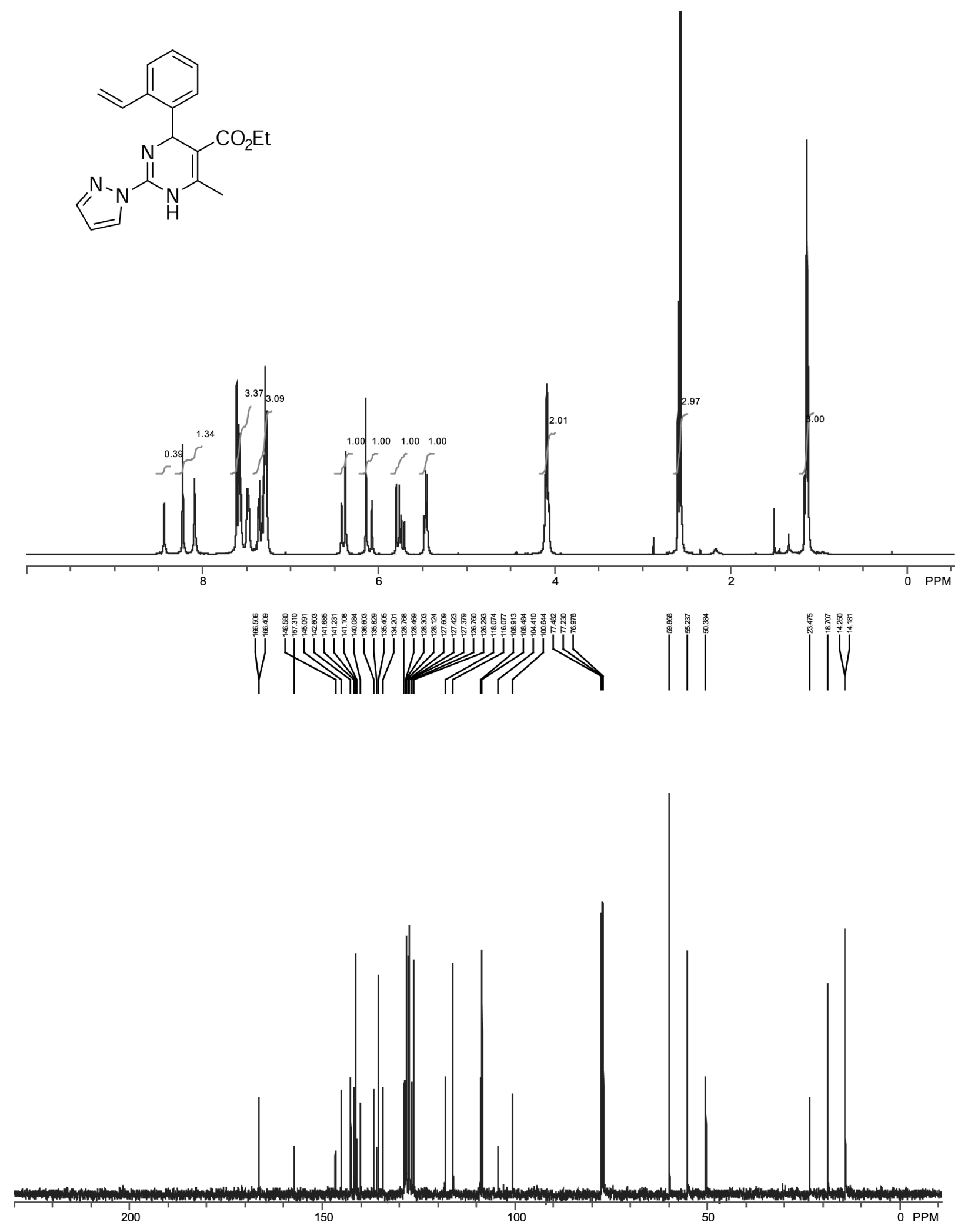
4-Isopropyl-6-methyl-2-pyrazol-1-yl-1,4-dihydropyrimidine-5-carboxylic acid benzyl ester (Compound 18c).
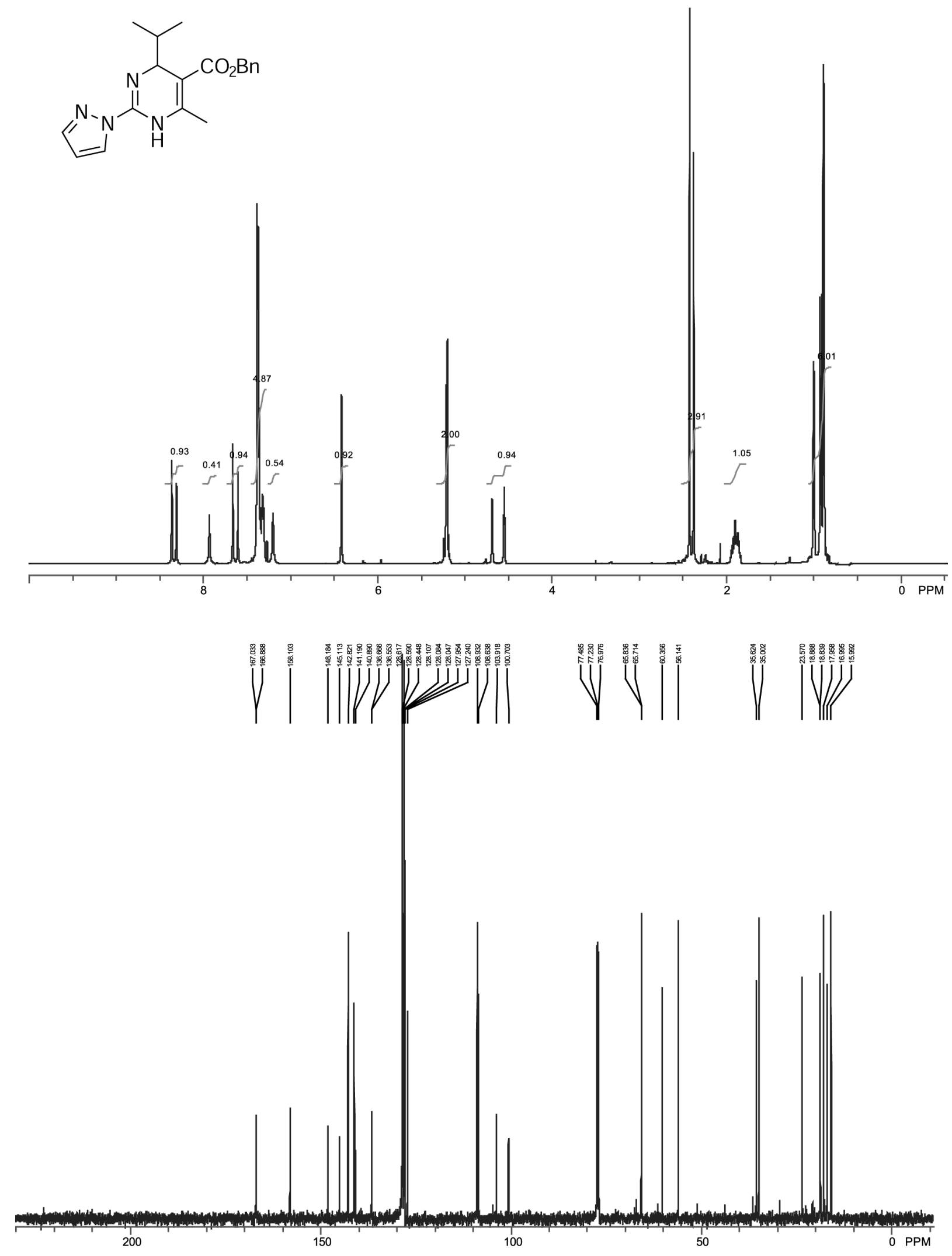
Supporting Information; Appendix 1: Nilsson and Overman 6-Methyl-4-propyl-2-pyrazol-1-yl-1,4-dihydropyrimidine-5-carboxylic acid benzyl ester (Compound 18d).
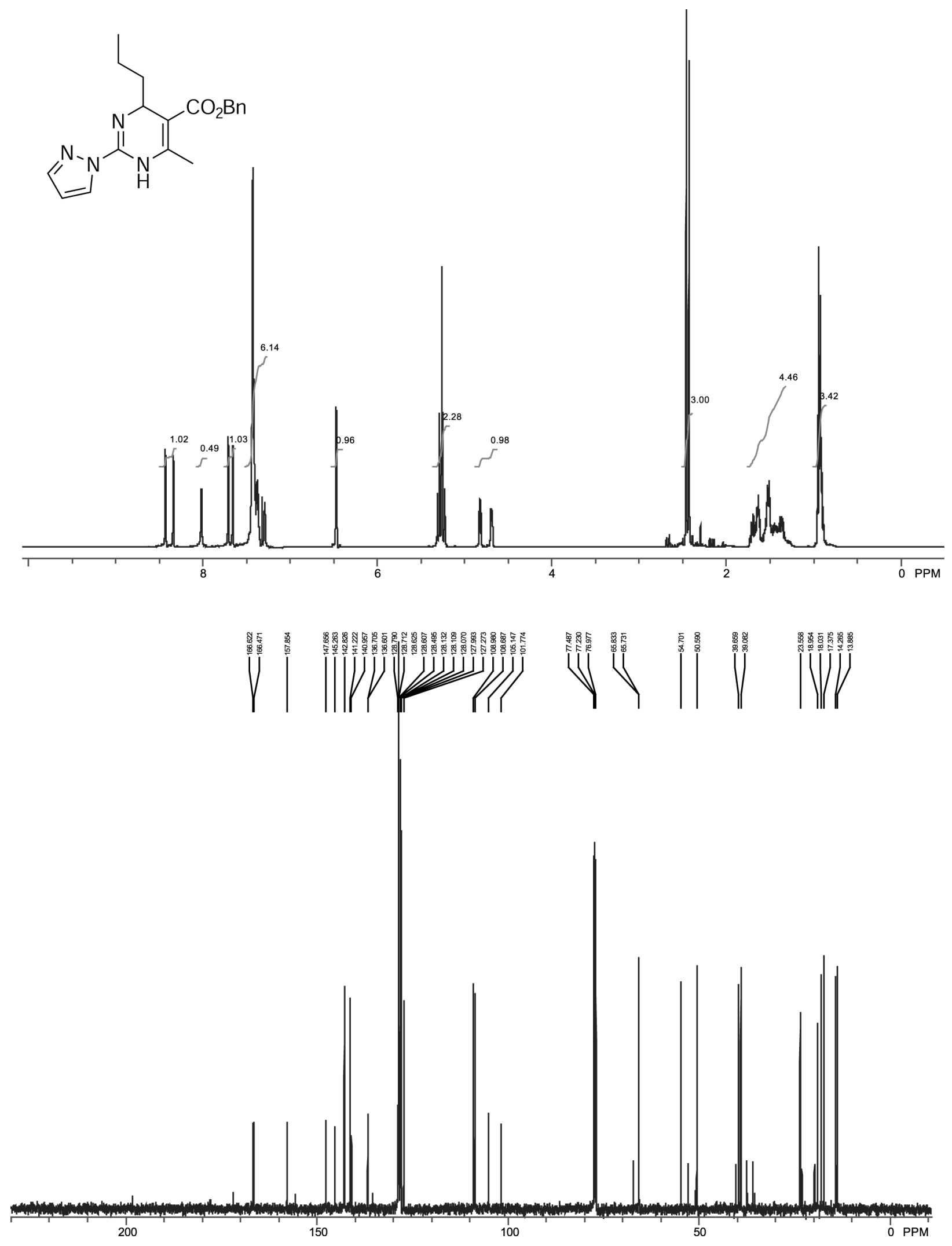
Supporting Information; Appendix 1: Nilsson and Overman 4-Cyclohexyl-6-methyl-2-pyrazol-1-yl-1,4-dihydropyrimidine-5-carboxylic acid benzyl ester (Compound 18e).
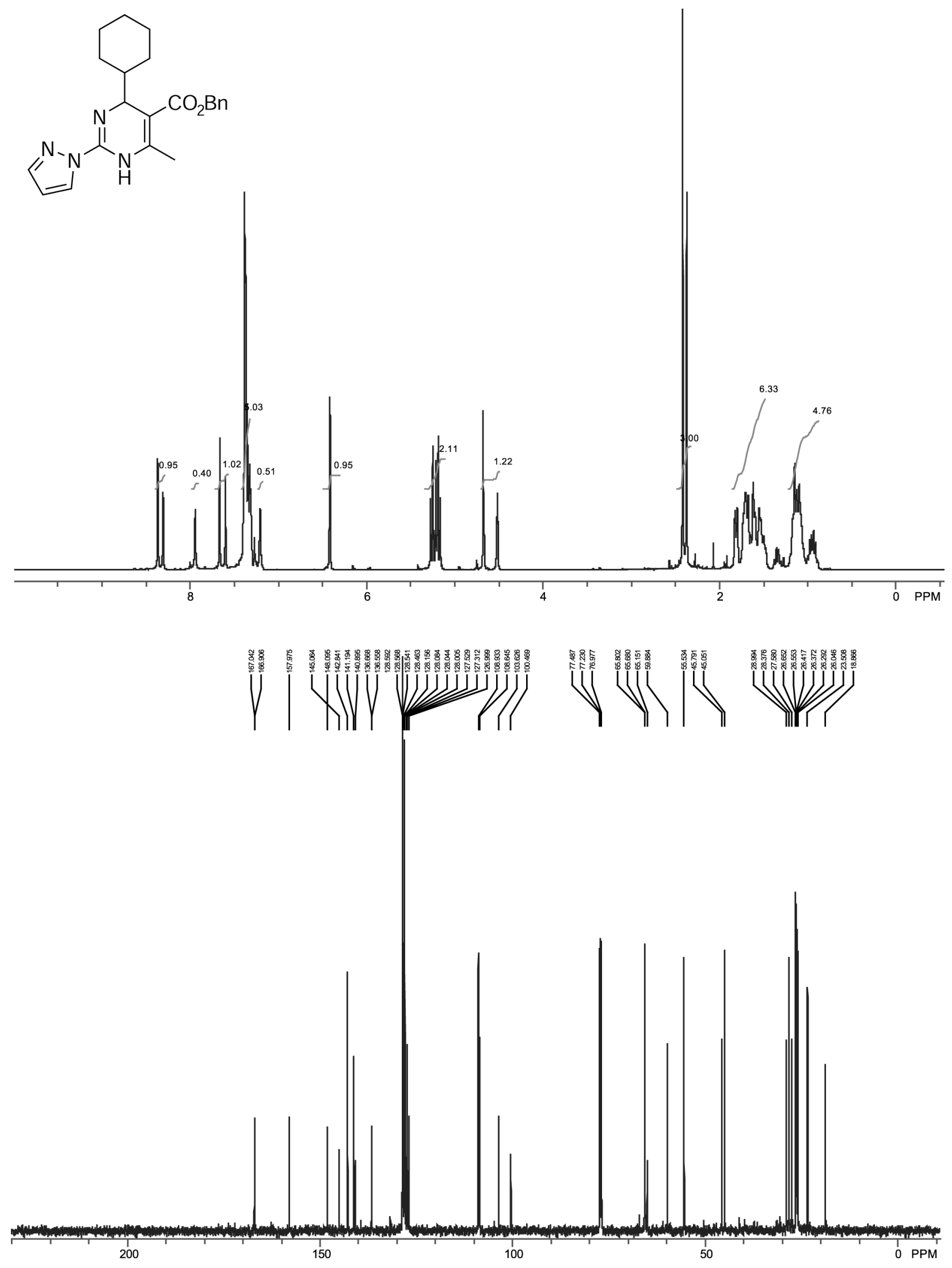

6-Methyl-4-(3-nitrophenyl)-2-pyrazol-1-yl-1,4-dihydropyrimidine-5-carboxylic acid ethyl ester (Compound 18f).
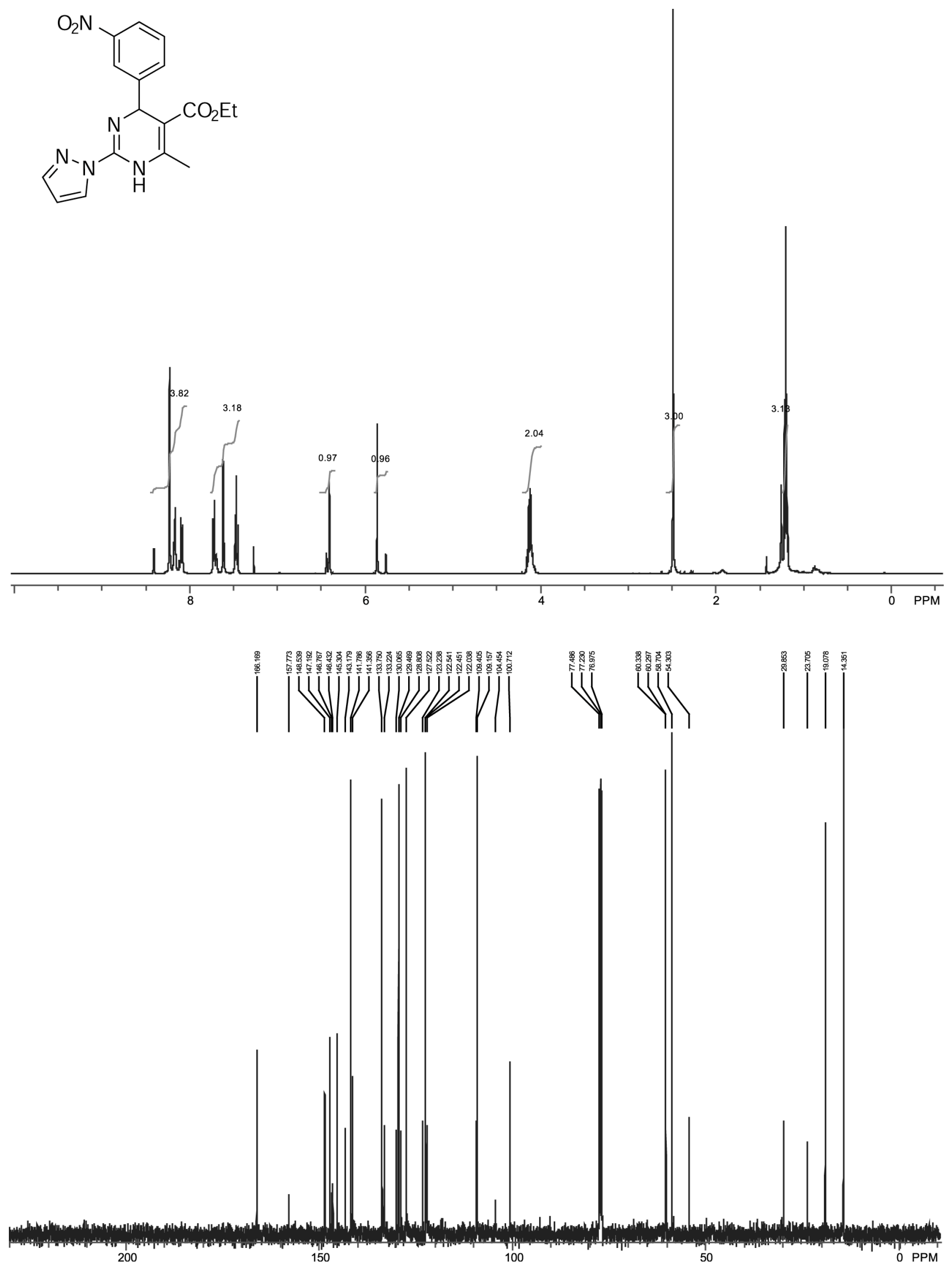

4-Methyl-6-phenyl-2-pyrazol-1-yl-6H-pyrimidine-1,5-dicarboxylic acid 1-tert-butyl ester 5-ethyl ester (Compound 19a).

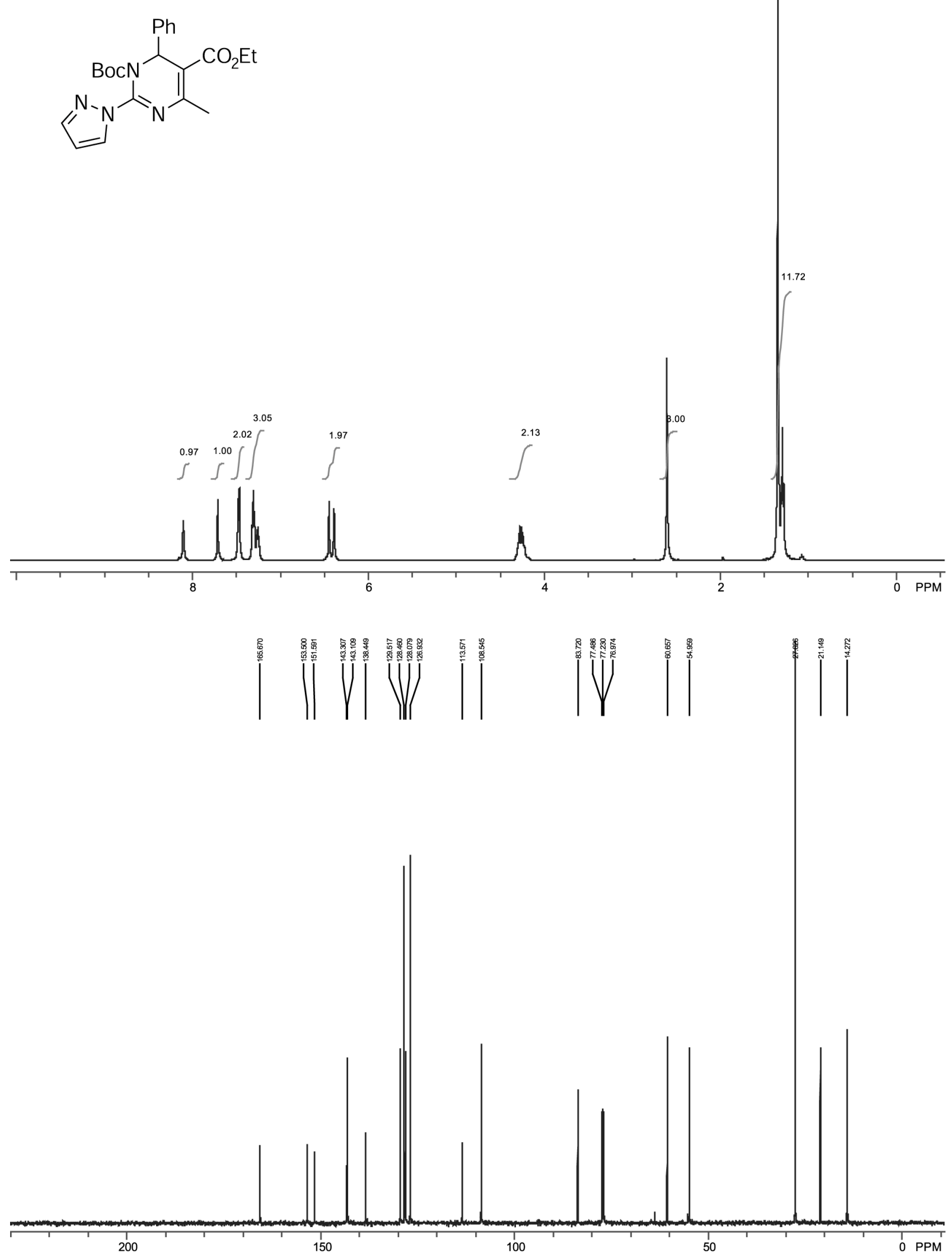


4-Methyl-2-pyrazol-1-yl-6-(2-vinylphenyl)-6H-pyrimidine-1,5-dicarboxylic acid 1-tert-butyl ester 5-ethyl ester (Compound 19b).<smiles></smiles>
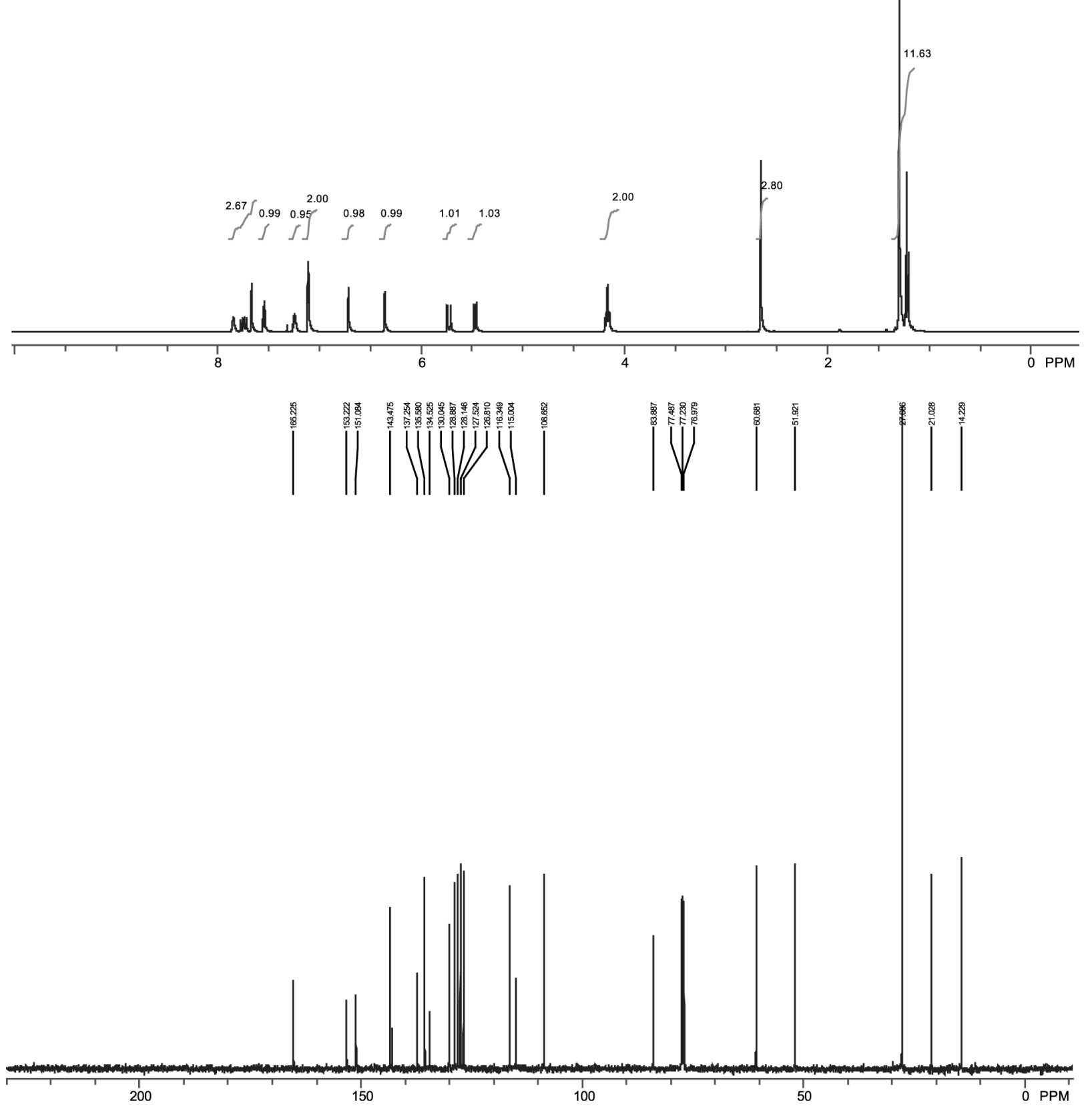
Supporting Information; Appendix 1: Nilsson and Overman 6-Isopropyl-4-methyl-2-pyrazol-1-yl-6H-pyrimidine-1,5-dicarboxylic acid 5-benzyl ester 1-tertbutyl ester (Compound 19c).<smiles></smiles>
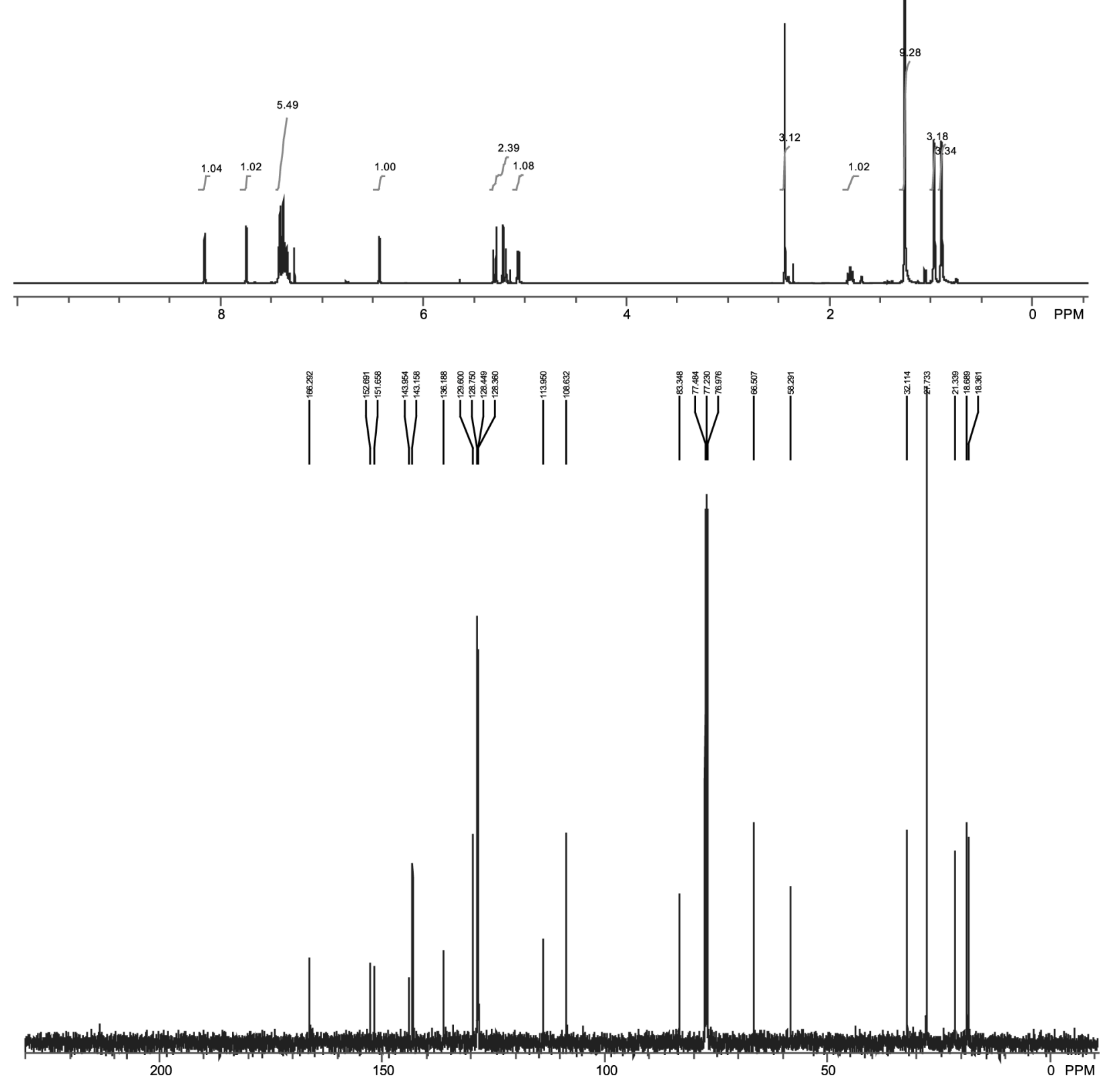

Methyl-6-propyl-2-pyrazol-1-yl-6H-pyrimidine-1,5-dicarboxylic acid 5-benzyl ester 1-tert-butyl ester (Compound 19d).<smiles>CCCC1C(C(=O)OCc2ccccc2)=C(C)N=C(n2cccn2)N1C(=O)OCc1ccccc1</smiles>
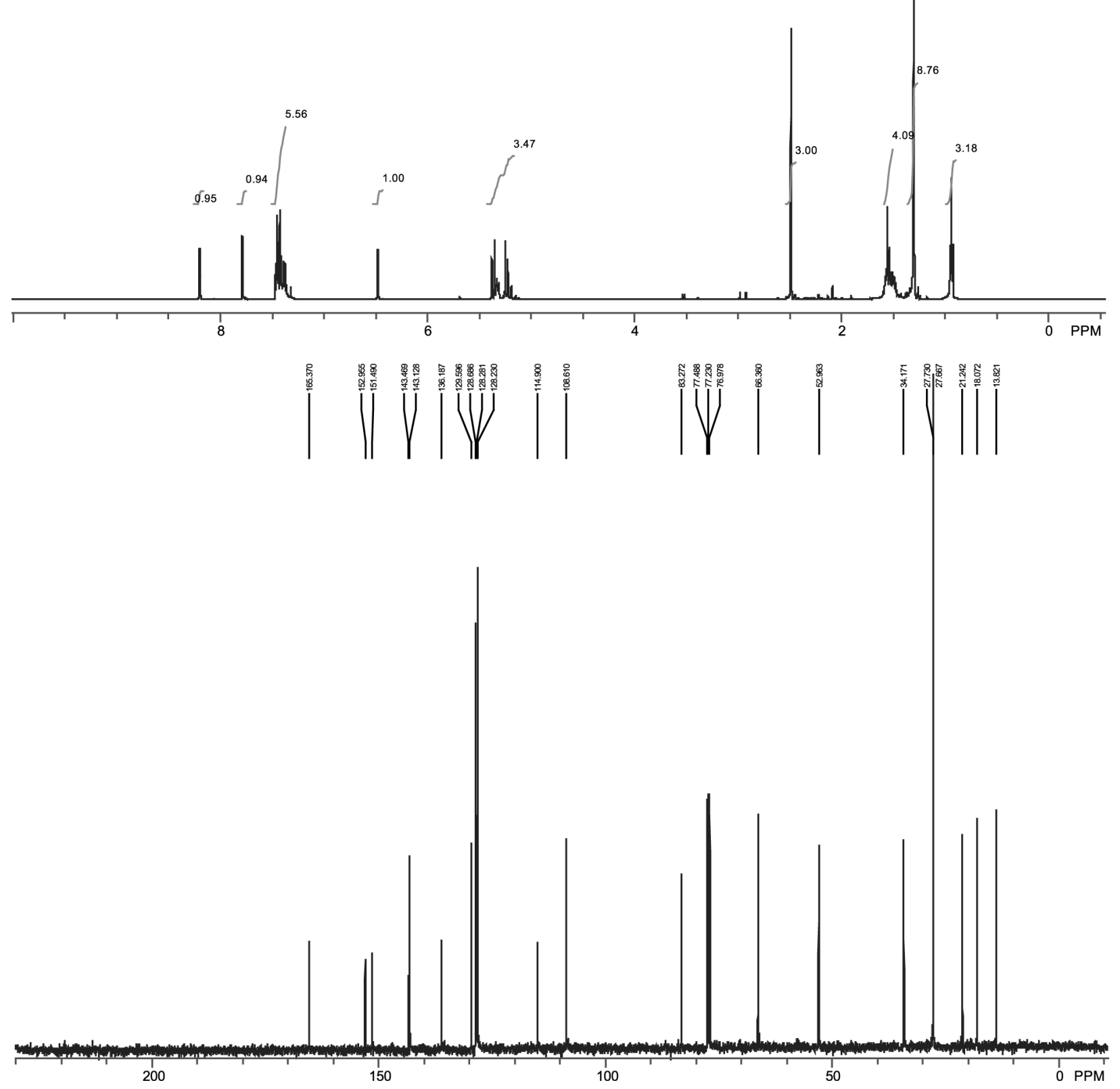
Supporting Information; Appendix 1: Nilsson and Overman 6-Cyclohexyl-4-methyl-2-pyrazol-1-yl-6H-pyrimidine-1,5-dicarboxylic acid 5-benzyl ester 1-tertbutyl ester (Compound 19e).<smiles>CC1=C(C(=O)OCc2ccccc2)C(C2CCCCC2)N(C(=O)c2ccccc2)C(n2cccn2)=N1</smiles>
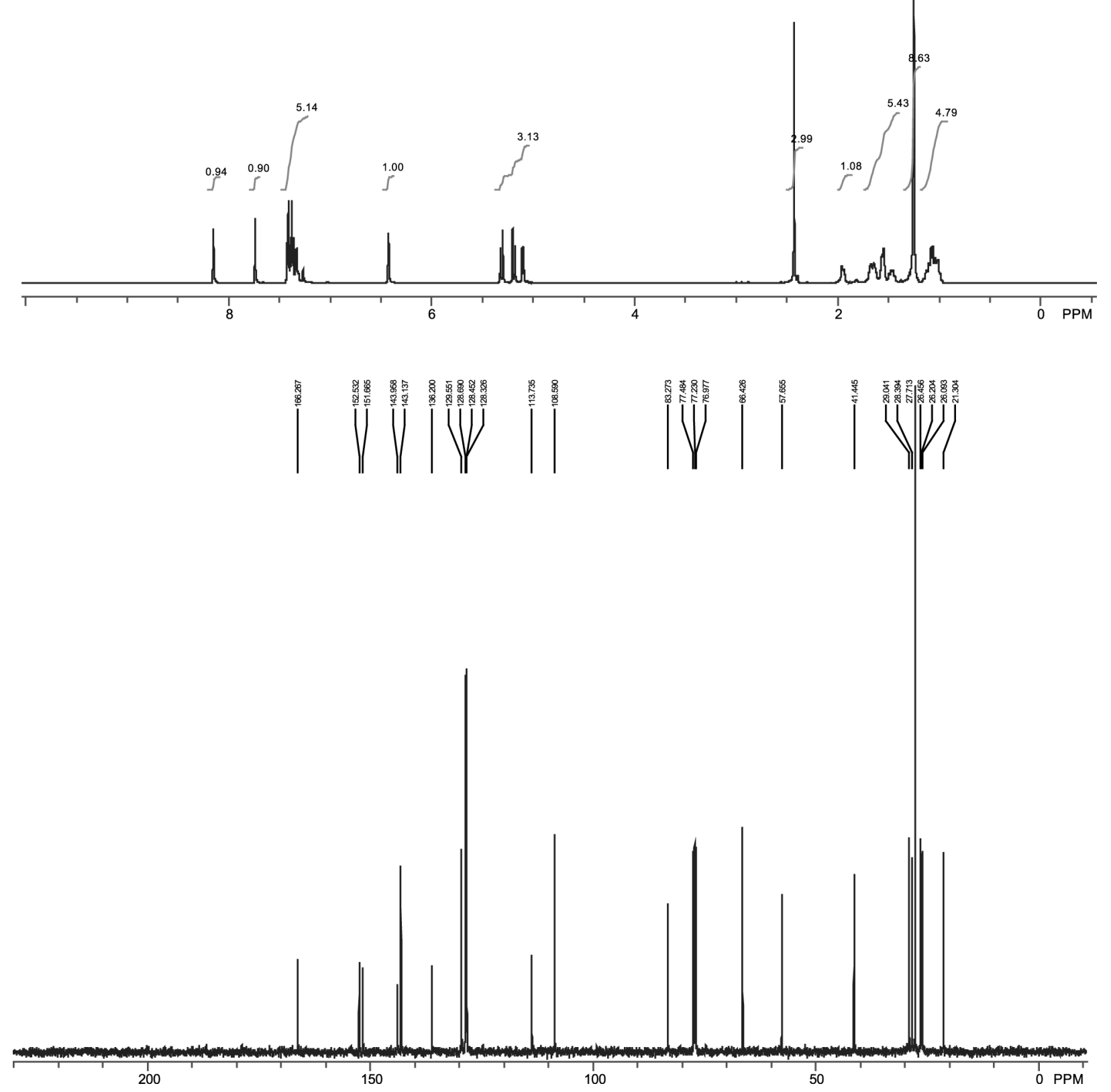
4-Methyl-6-(3-nitrophenyl)-2-pyrazol-1-yl-6H-pyrimidine-1,5-dicarboxylic acid 1-tert-butyl ester 5-ethyl ester (Compound 19f).<smiles>CCOC(=O)C1=C(C)N=C(n2cccn2)N(C(=O)c2ccccc2)C1c1cccc([N+](=O)[O-])c1</smiles>
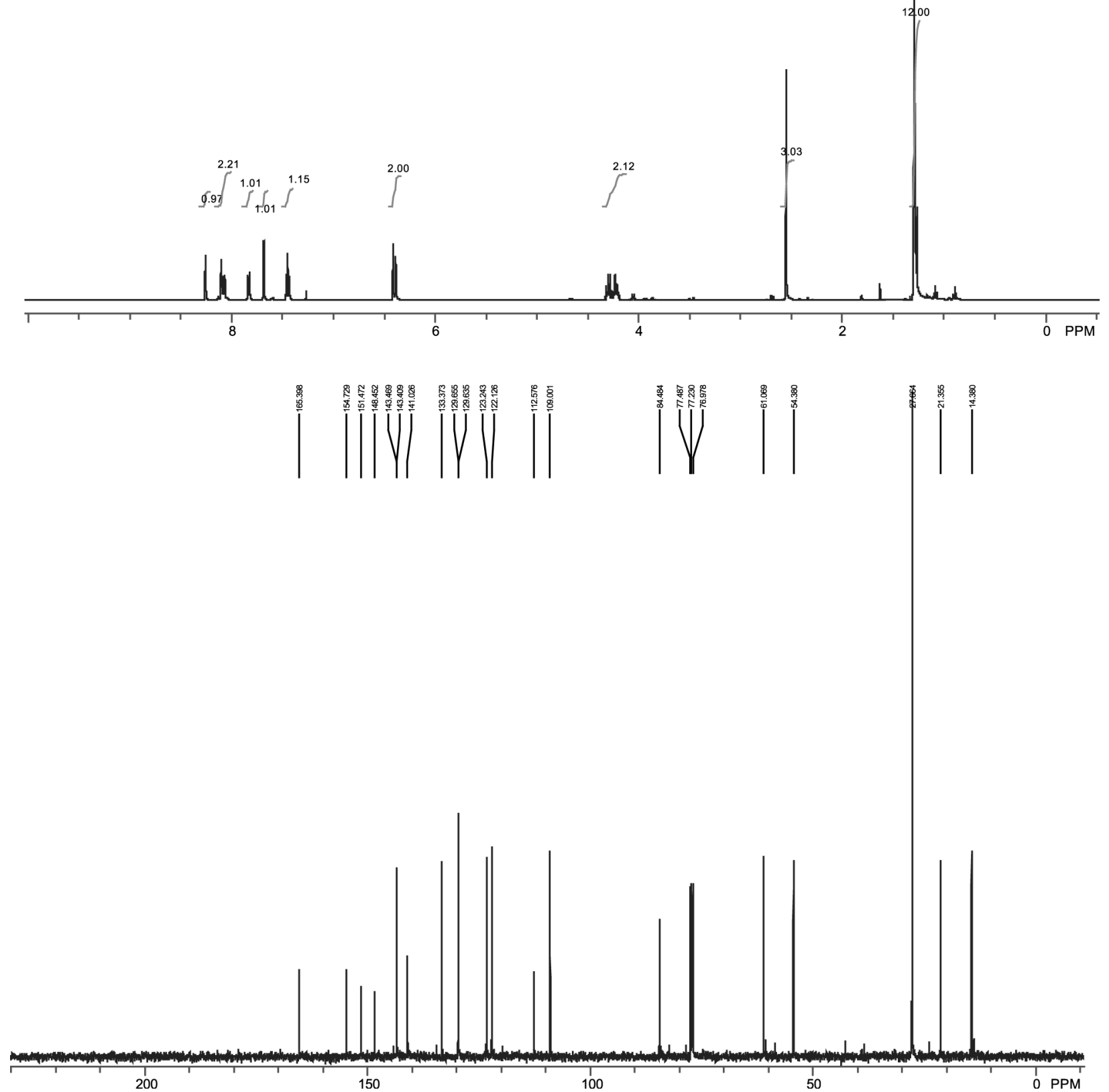
2-Amino-4-methyl-6-phenyl-6H-pyrimidine-1,5-dicarboxylic acid 1-tert-butyl ester 5-ethyl ester (Compound 16a).

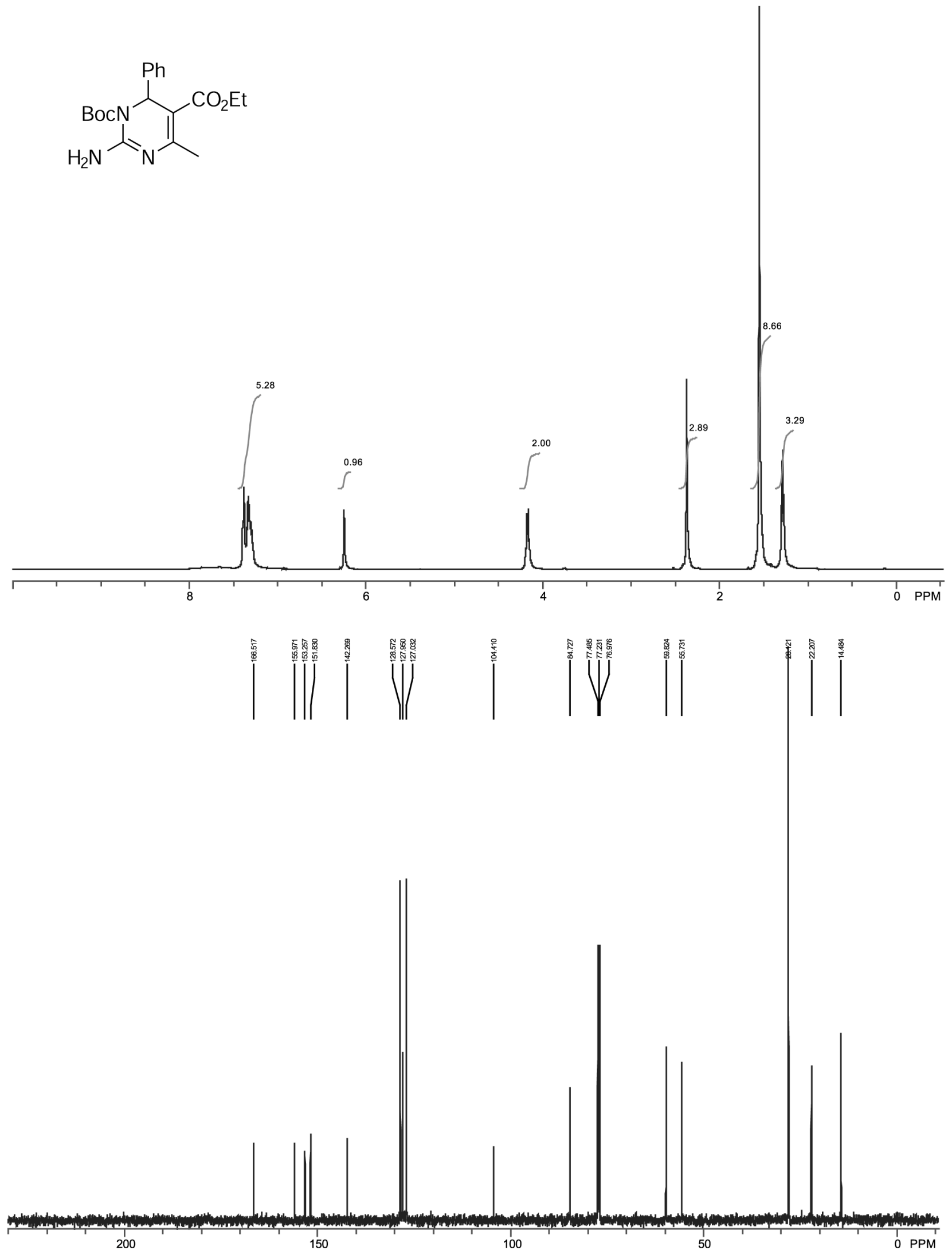


Supporting Information; Appendix 1: Nilsson and Overman

2-Amino-4-methyl-6-(2-vinylphenyl)-6H-pyrimidine-1,5-dicarboxylic acid 1-tert-butyl ester 5ethyl ester (Compound 16b).<smiles>C=Cc1ccccc1C1C(C(=O)OCC)=C(C)N=C(N)N1C(=O)OCc1ccccc1</smiles>
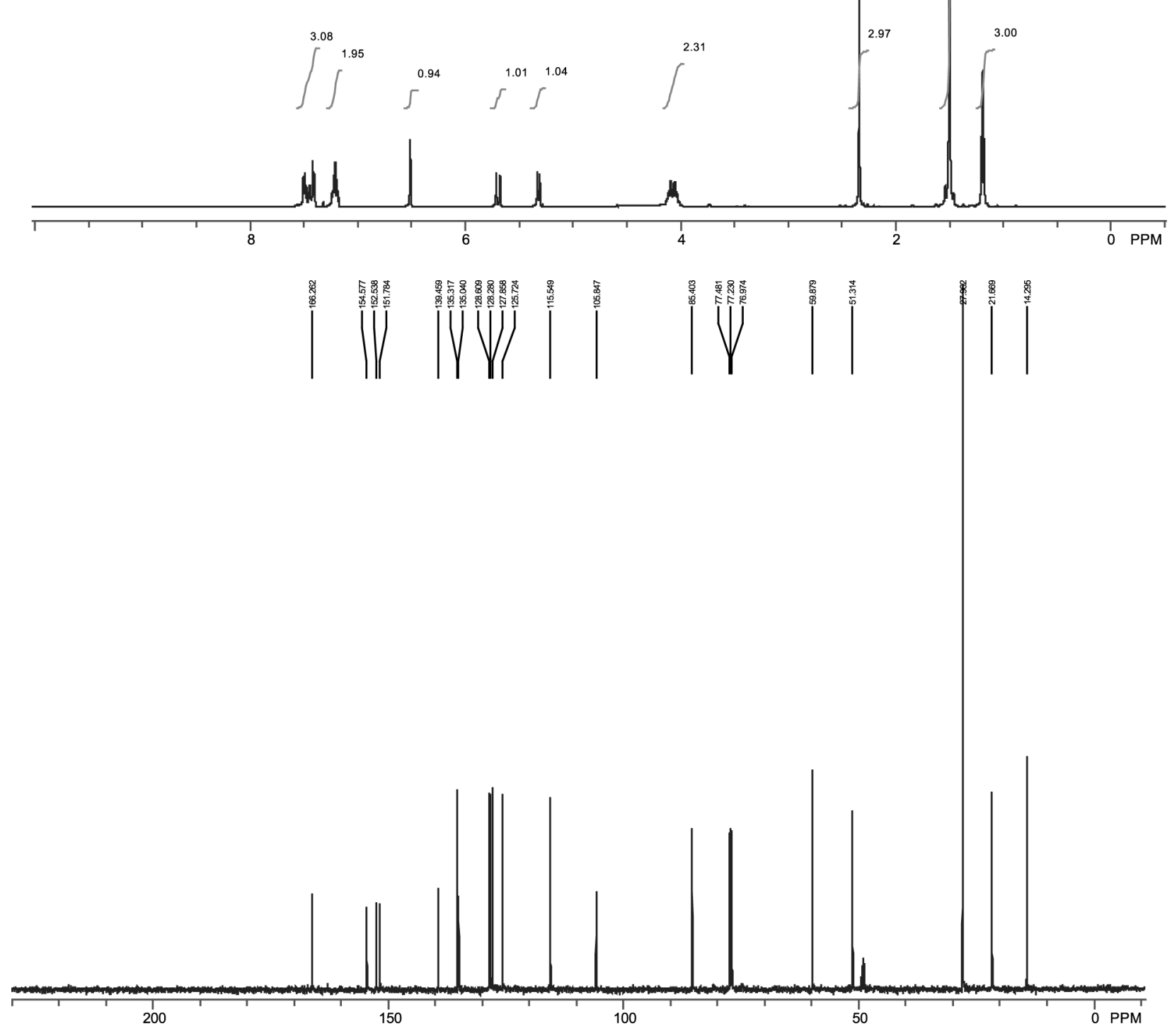
2-Amino-6-isopropyl-4-methyl-6H-pyrimidine-1,5-dicarboxylic acid 5-benzyl ester 1-tert-butyl ester (Compound 16c).
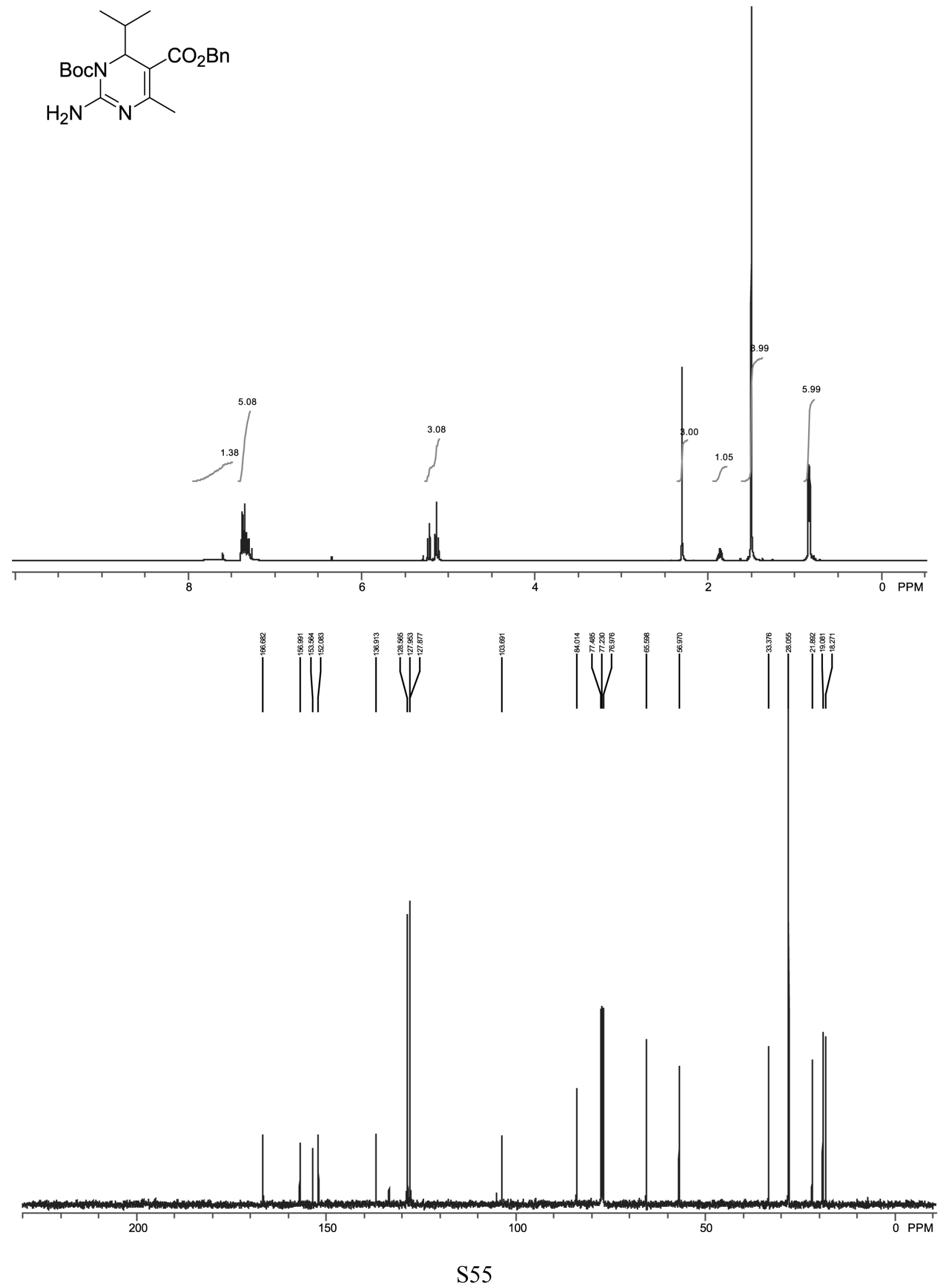
Supporting Information; Appendix 1: Nilsson and Overman 2-Amino-4-methyl-6-propyl-6H-pyrimidine-1,5-dicarboxylic acid 5-benzyl ester 1-tert-butyl ester (Compound 16d).

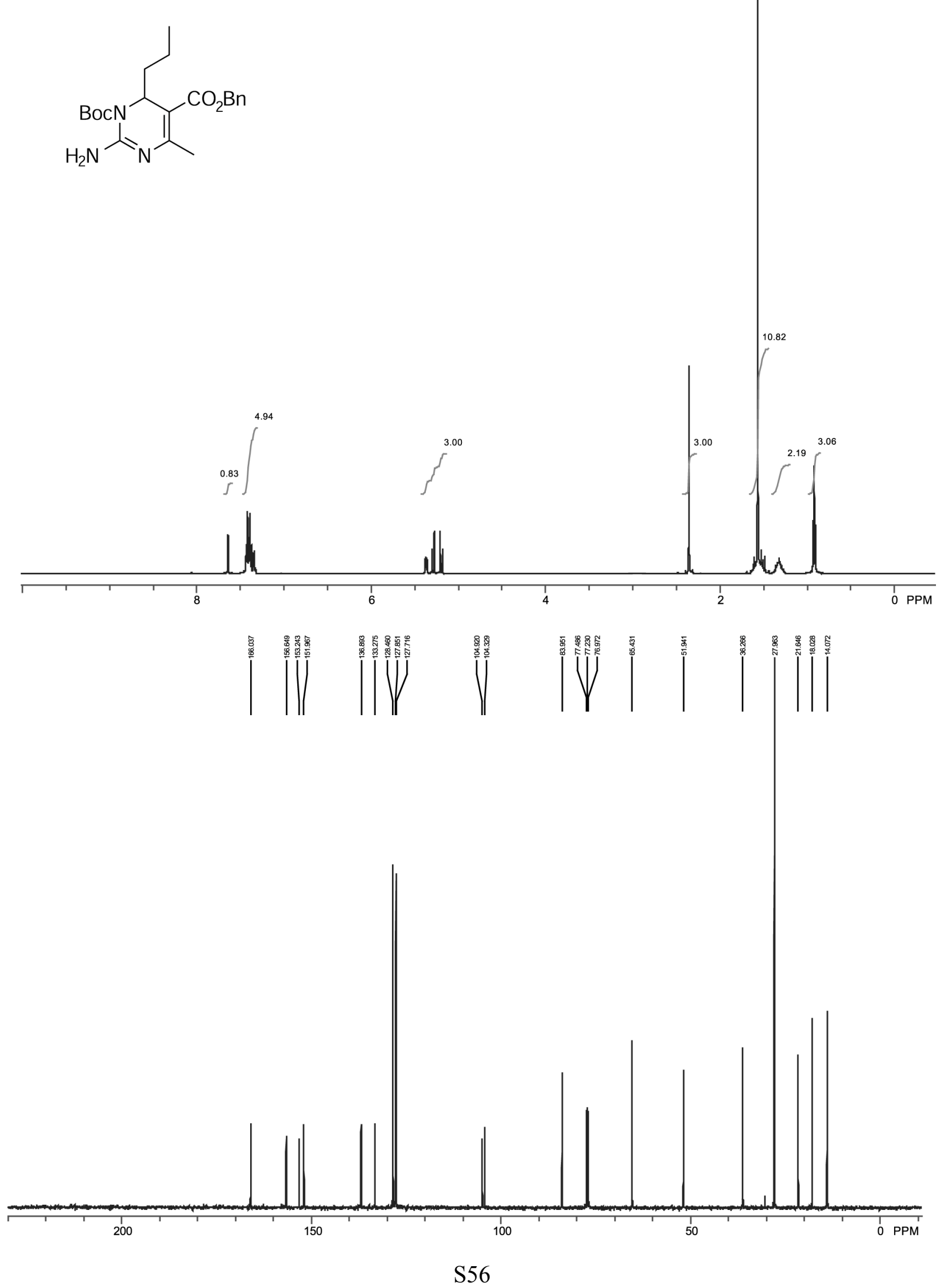


2-Amino-6-cyclohexyl-4-methyl-6H-pyrimidine-1,5-dicarboxylic acid 5-benzyl ester 1-tert-butyl ester (Compound 16e).<smiles>CC1=C(C(=O)OCc2ccccc2)C(C2CCCCC2)N(C(=O)OCc2ccccc2)C(N)=N1</smiles>
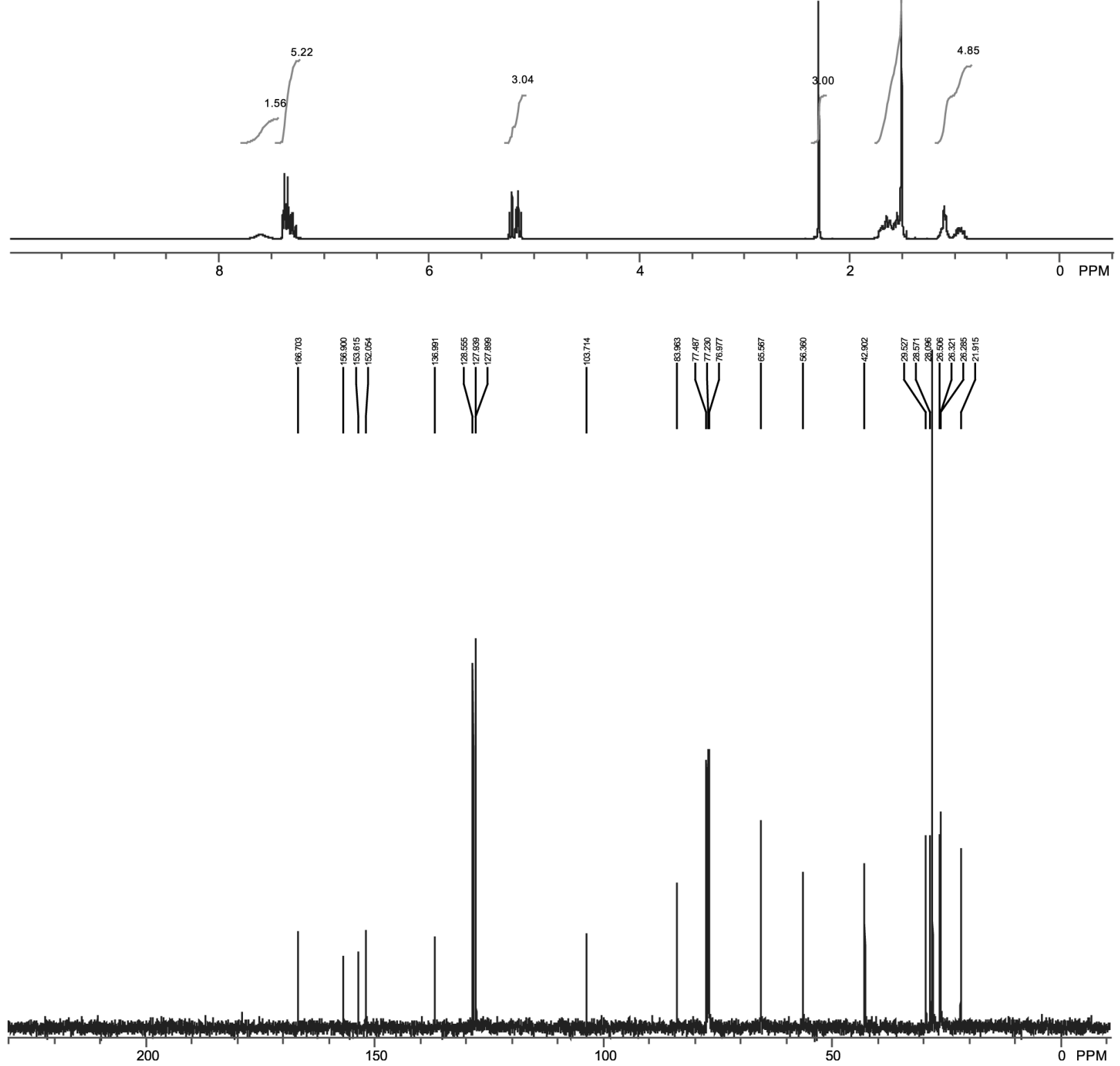
2-Amino-4-methyl-6-(3-nitro)-6H-pyrimidine-1,5-dicarboxylic acid 1-tert-butyl ester 5-ethyl ester (Compound 16f).
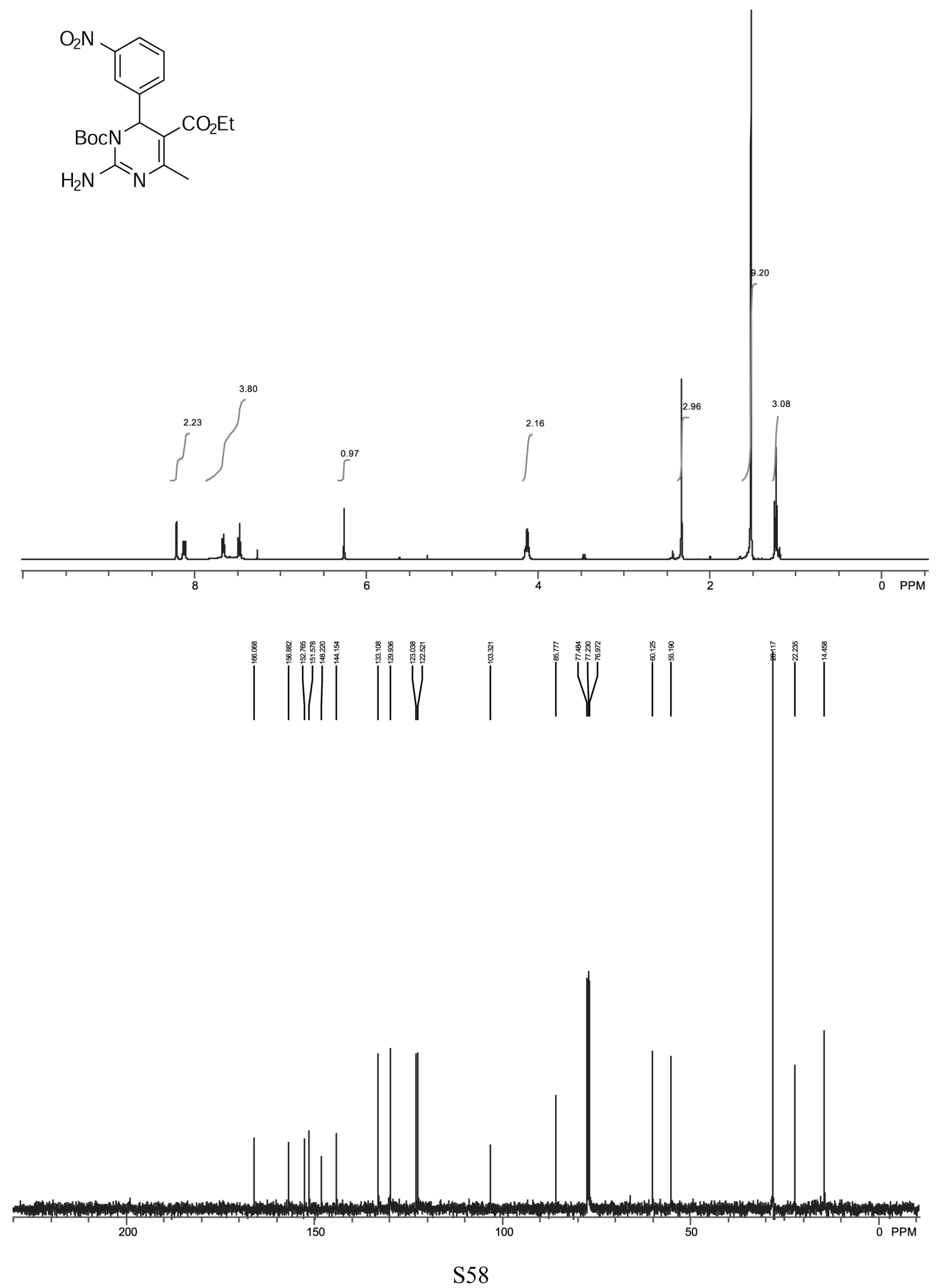
Supporting Information; Appendix 1: Nilsson and Overman 5-Ethoxycarbonyl-6-methyl-4-phenyl-3,4-dihydro-1 $H$-pyrimidin-2-ylidene-ammonium trifluoroacetate (Compound 10a).

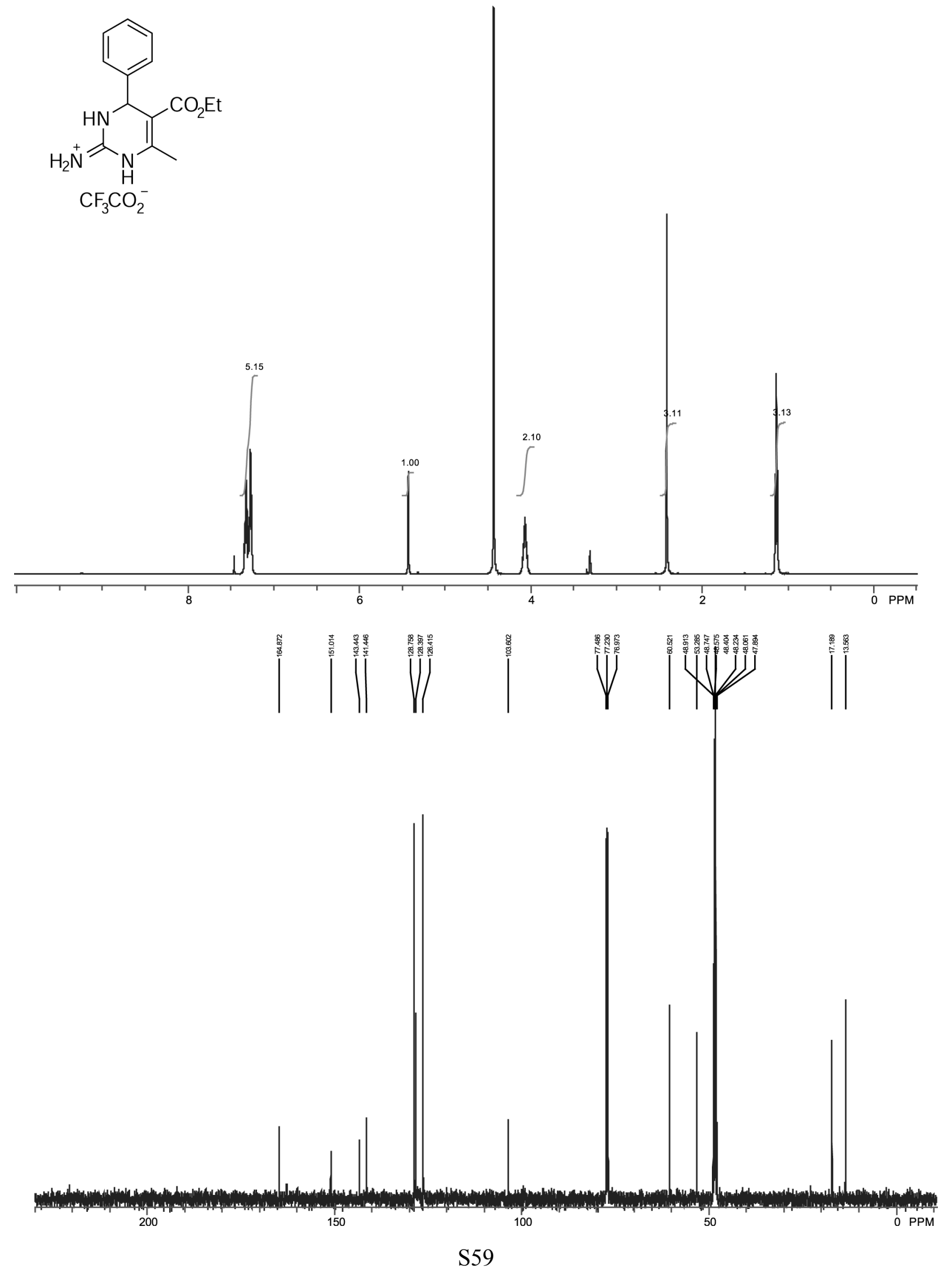


Supporting Information; Appendix 1: Nilsson and Overman 5-Ethoxycarbonyl-6-methyl-4-(2-vinylphenyl)-3,4-dihydro-1 $H$-pyrimidin-2-ylidene-ammonium trifluoroacetate (Compound 10b).

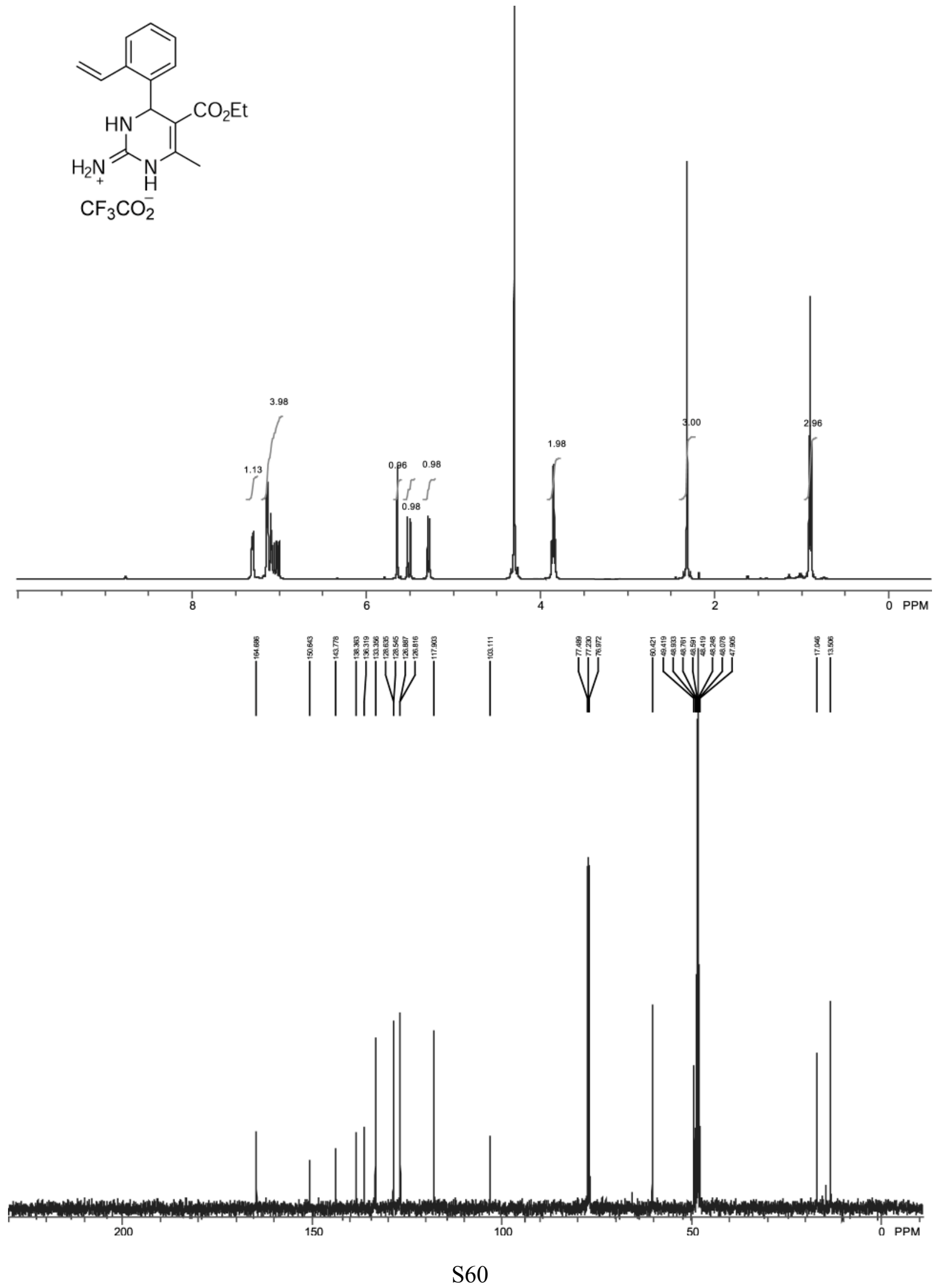


5-Benzyloxycarbonyl-4-isopropyl-6-methyl-3,4-dihydro-1H-pyrimidin-2-ylidene-ammonium trifluoroacetate (Compound 10c).

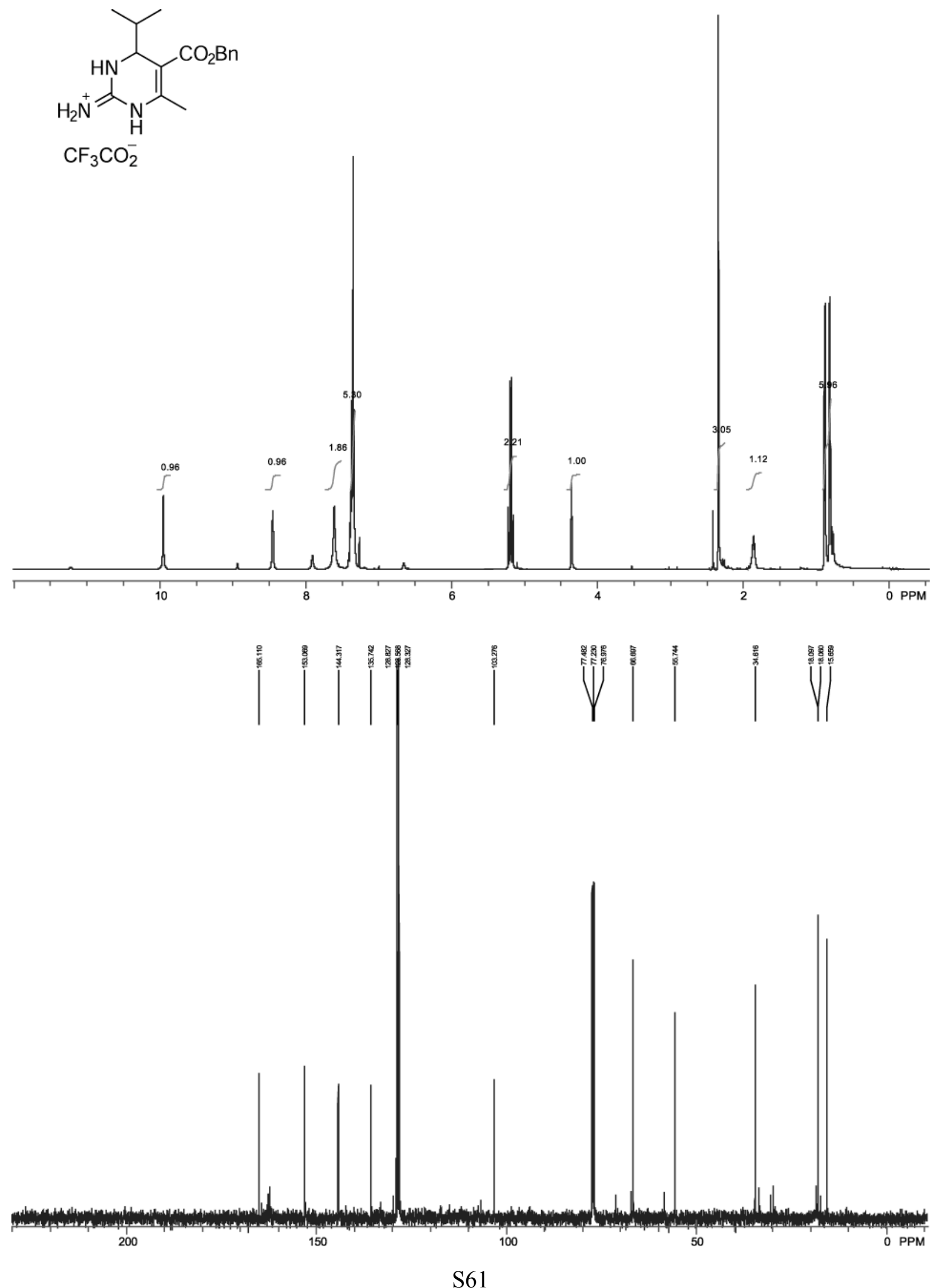


Supporting Information; Appendix 1: Nilsson and Overman 5-Benzyloxycarbonyl-6-methyl-4-propyl-3,4-dihydro-1 $H$-pyrimidin-2-ylidene-ammonium trifluoroacetate (Compound 10d).

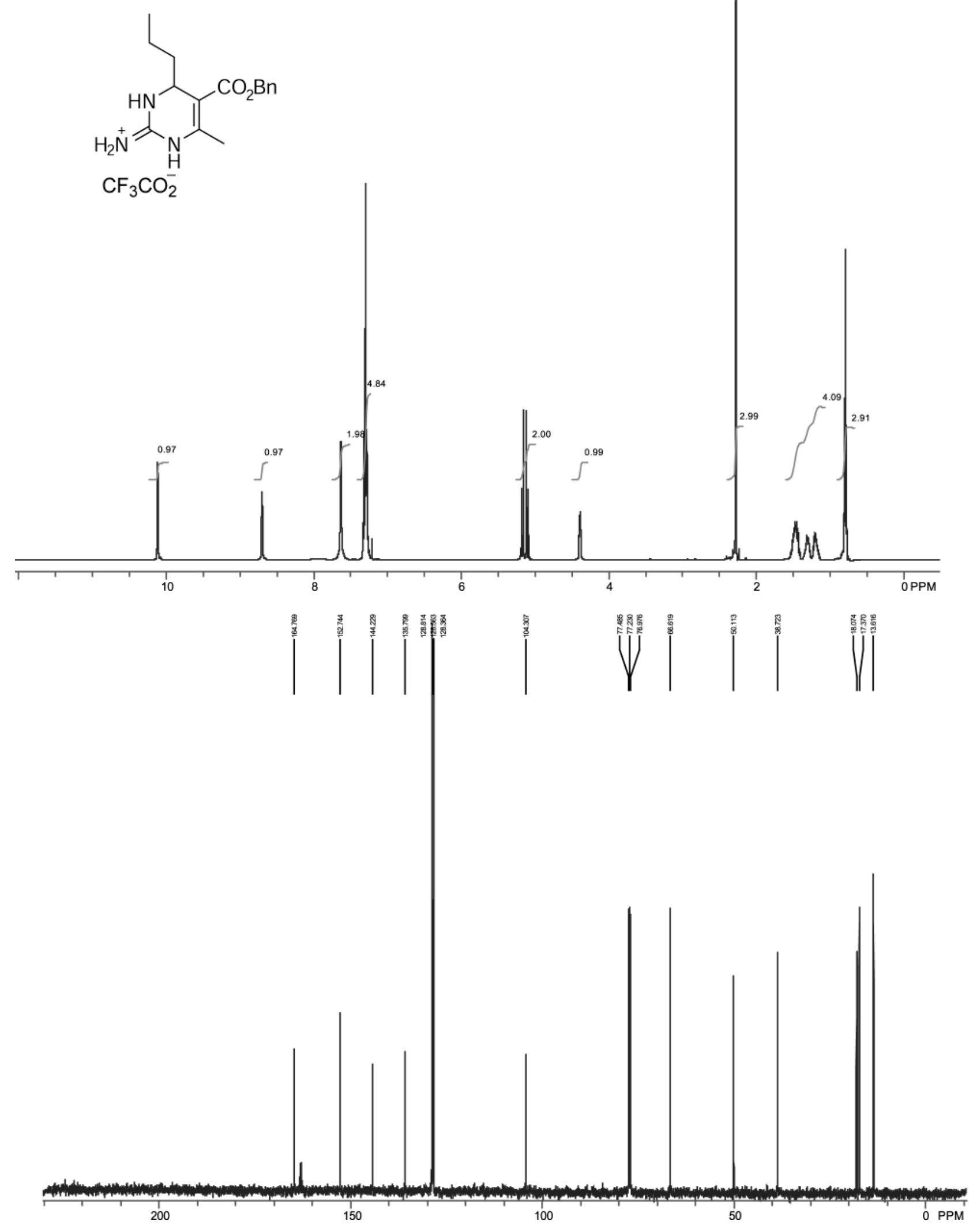


5-Benzyloxycarbonyl-4-cyclohexyl-6-methyl-3,4-dihydro-1 $H$-pyrimidin-2-ylidene-ammonium trifluoroacetate (Compound 10e).<smiles>CC1=C(C(=O)OC(F)(F)F)C(C2CCCCC2)NC(=[18O])N1</smiles>
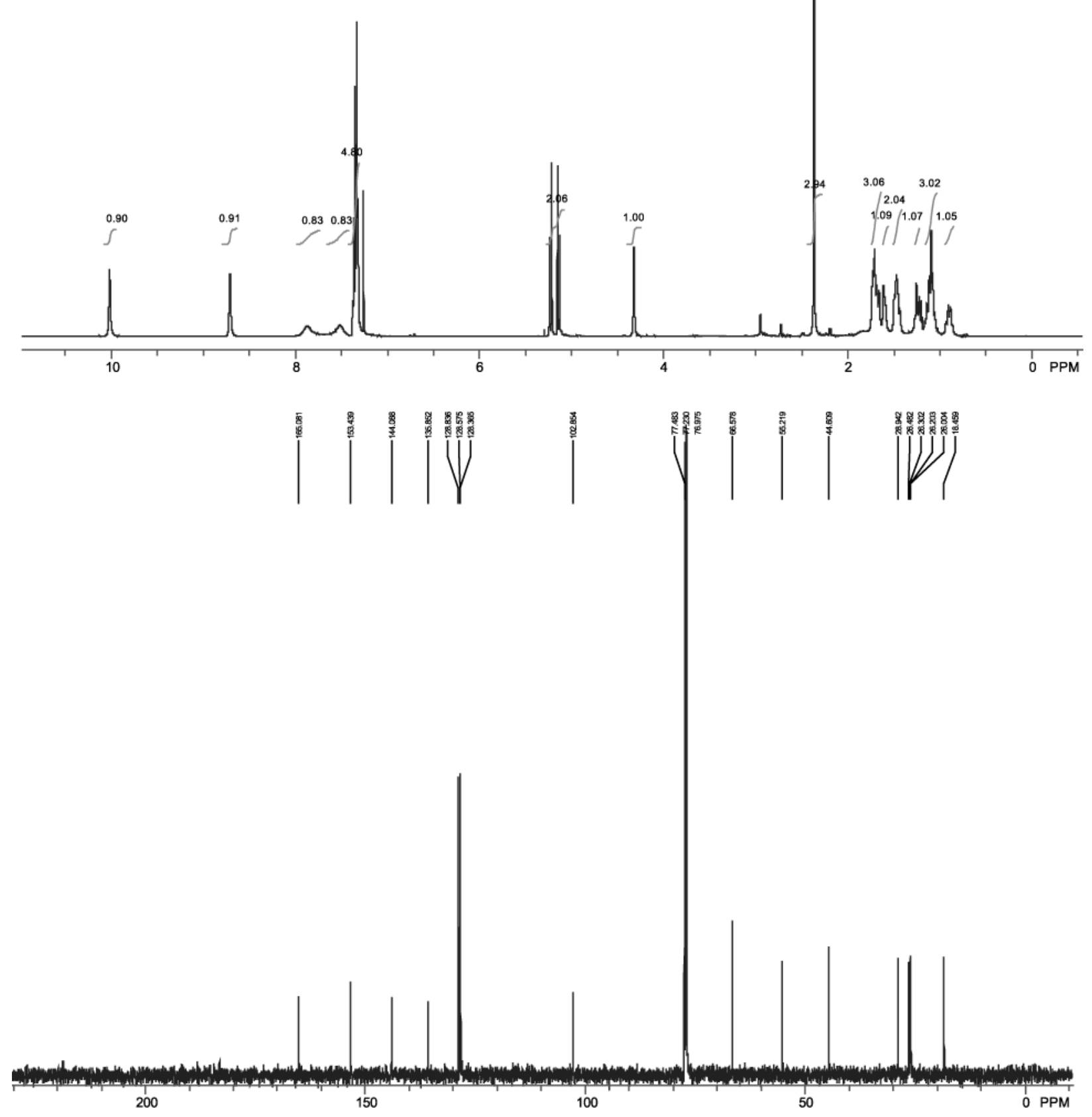
5-Ethoxycarbonyl-6-methyl-4-(3-nitrophenyl)-3,4-dihydro-1H-pyrimidin-2-ylidene-ammonium trifluoroacetate (Compound 10f).<smiles>CCOC(=O)C1=C(C)NC(=[NH2+])NC1c1cccc([N+](=O)[O-])c1</smiles>
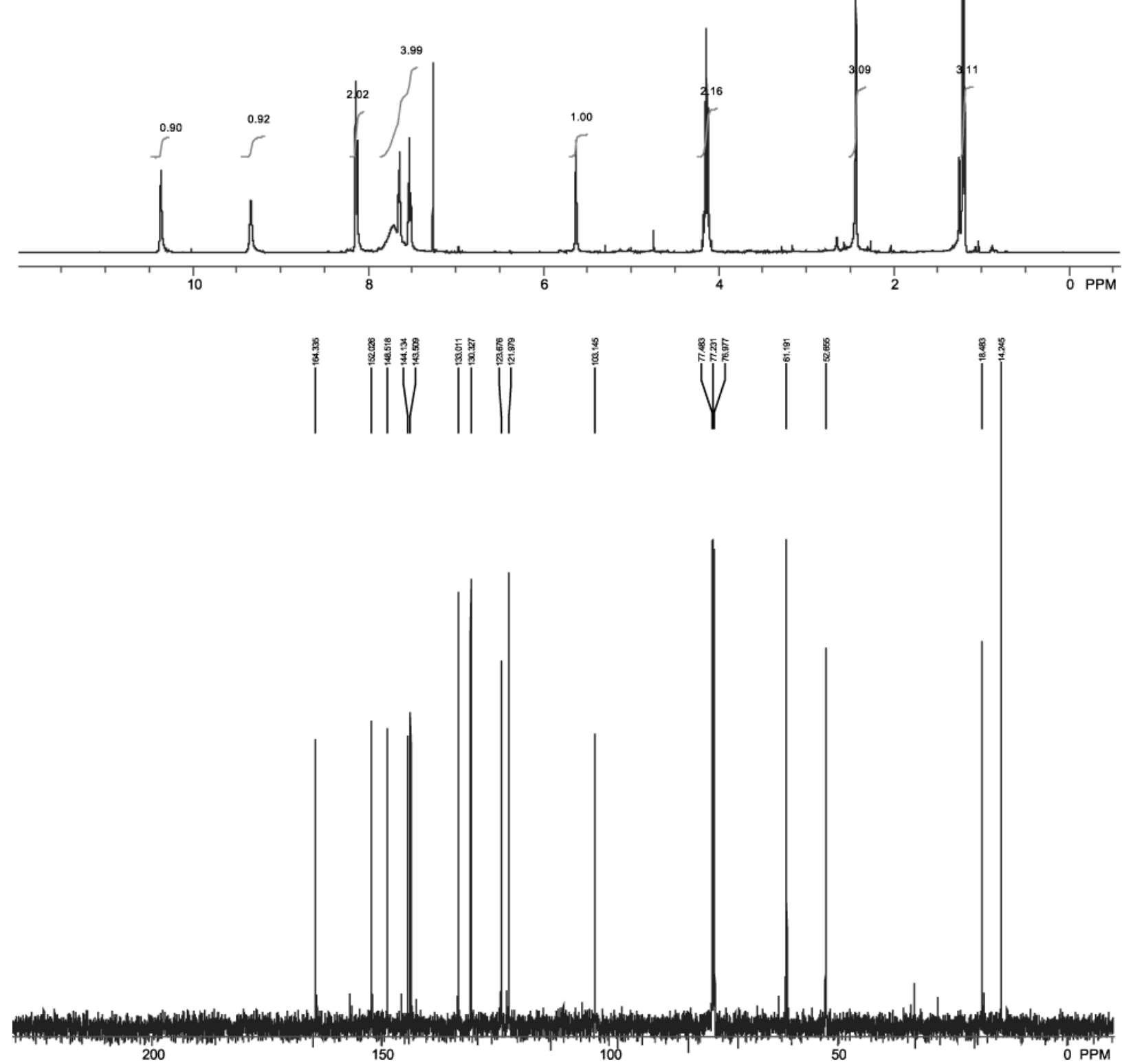
Supporting Information; Appendix 1: Nilsson and Overman 5-Ethoxycarbonyl-6-methyl-4-(4-nitrophenyl)-3,4-dihydro-1H-pyrimidin-2-ylidene-ammonium chloride (Compound 10i).<smiles>CCOC(=O)C1=C(C)NC(=[NH2+])NC1c1ccc([N+](=O)[O-])cc1</smiles>
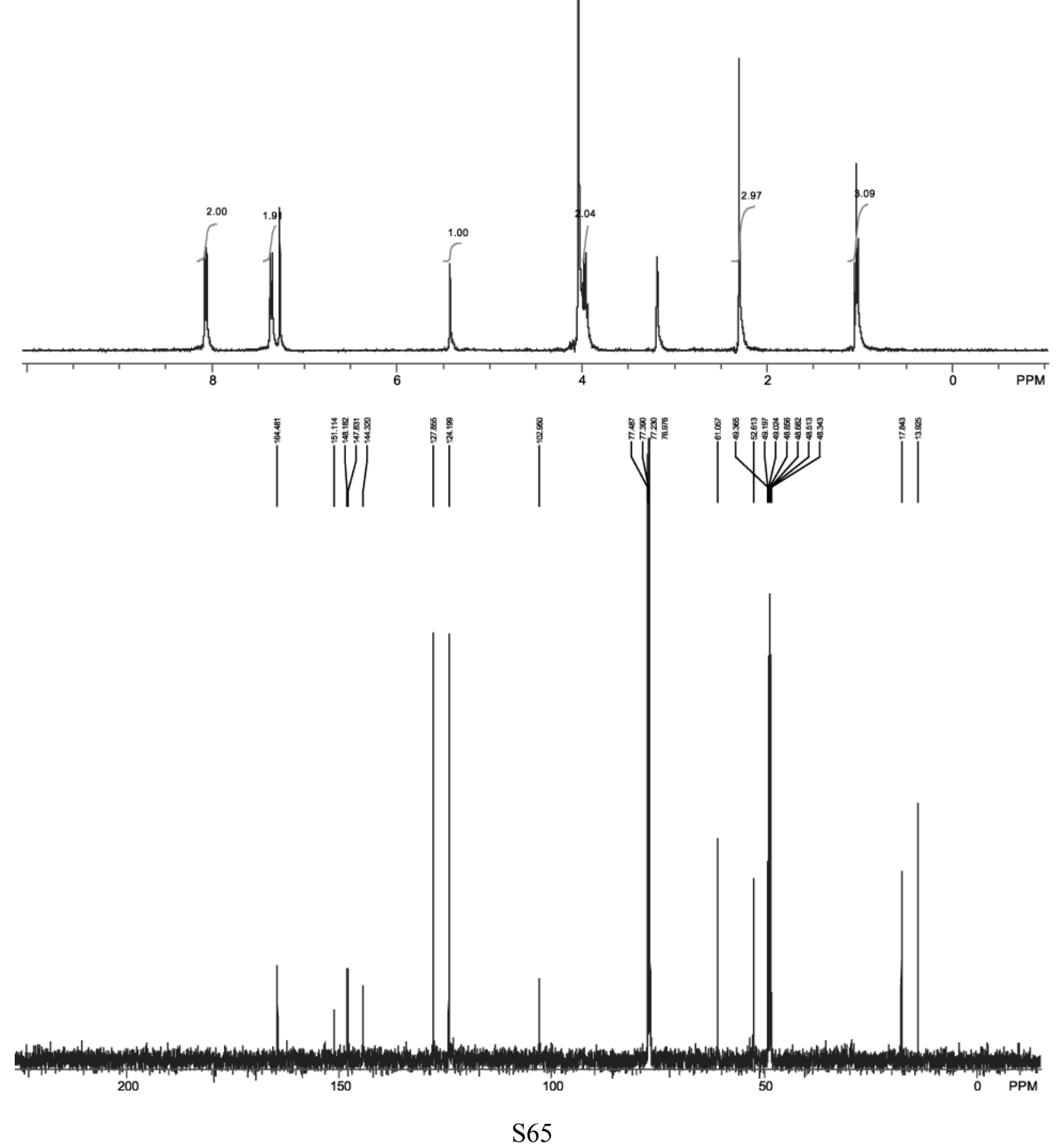
Supporting Information; Appendix 1: Nilsson and Overman 5-Benzyl-1,3-dimethyl-[1,3,5]triazinan-2-one (Compound 25).

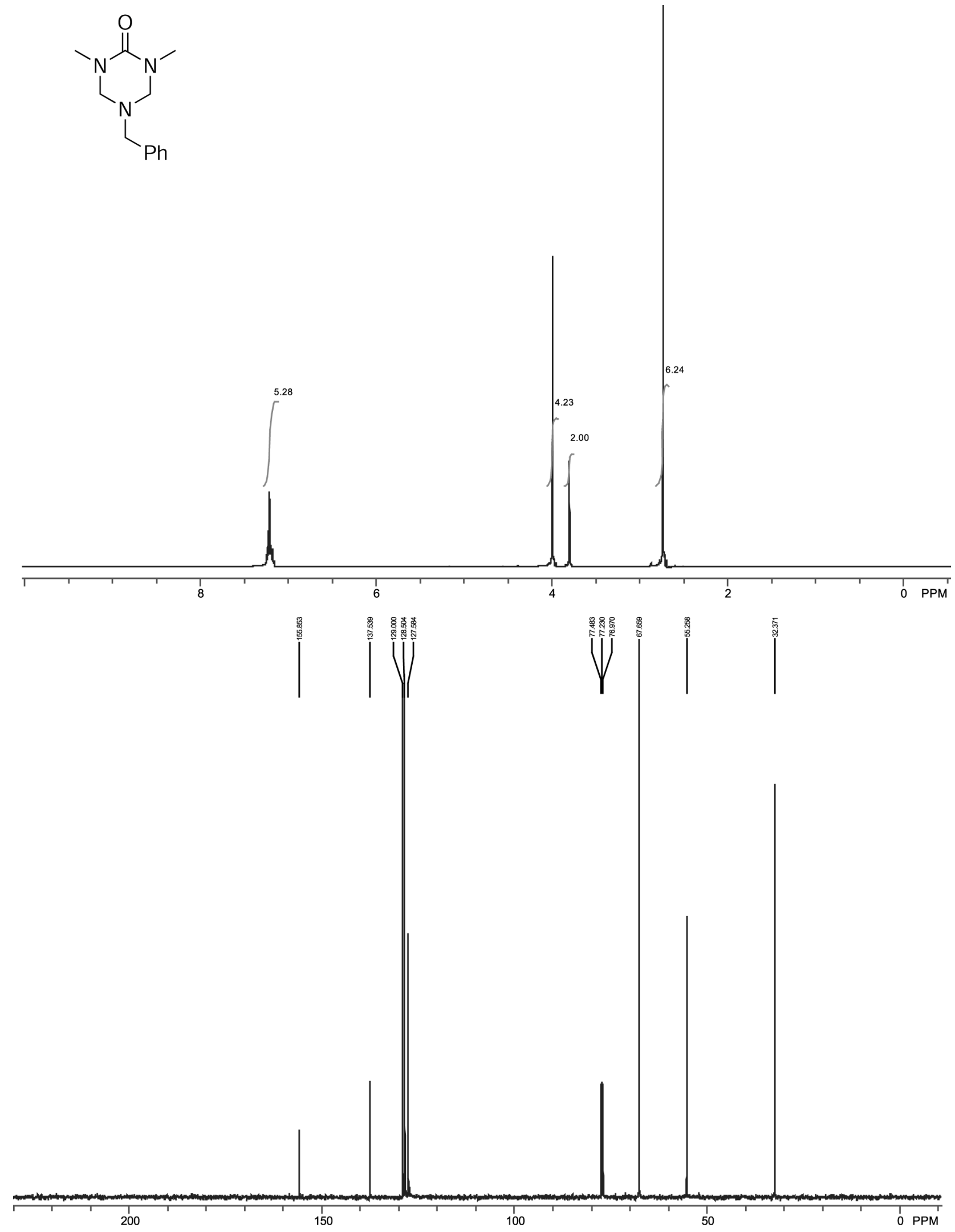


Supporting Information; Appendix 1: Nilsson and Overman 1,3-Dimethyl-[1,3,5]triazinan-2-one (Compound 26).<smiles>CN1CNCN(C)C1=O</smiles>
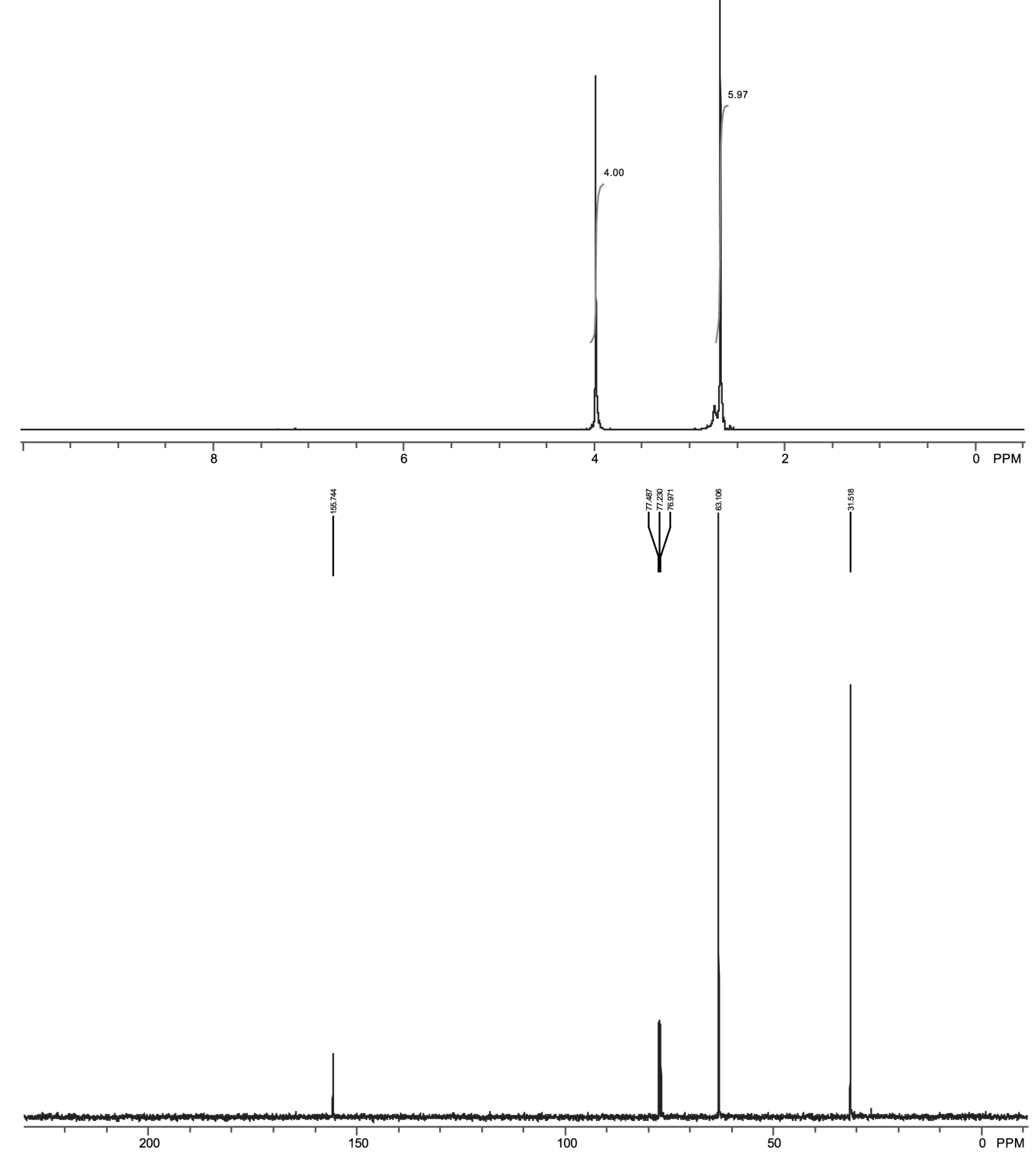
Supporting Information; Appendix 1: Nilsson and Overman

Tert-Butyloxycarbonylimino-[(3,5-dimethyl-4-oxo-[1,3,5]triazinan-1-yl)-methyl]-carbamic acid tert-butyl ester (Compound 28).<smiles>CN1CN(C(=NC(=O)OC(C)(C)C)C(C)(C)C)CN(C)C1=O</smiles>
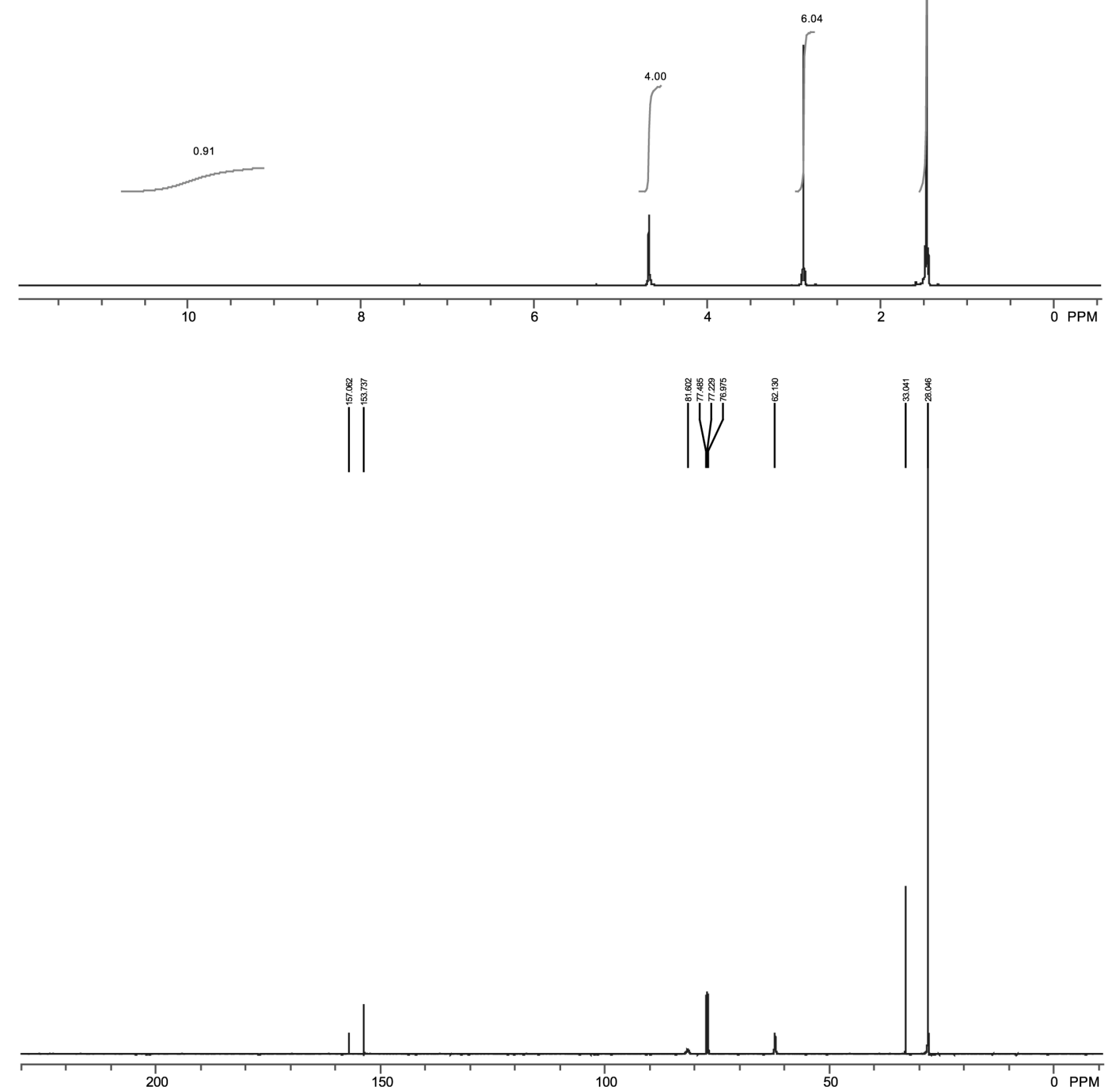
Supporting Information; Appendix 1: Nilsson and Overman 3,5-Dimethyl-4-oxo-[1,3,5] triazinane-1-carboxamidine TFA salt (Compound 20).<smiles>CN1CN(C(=N)N)CN(C)C1=O</smiles>
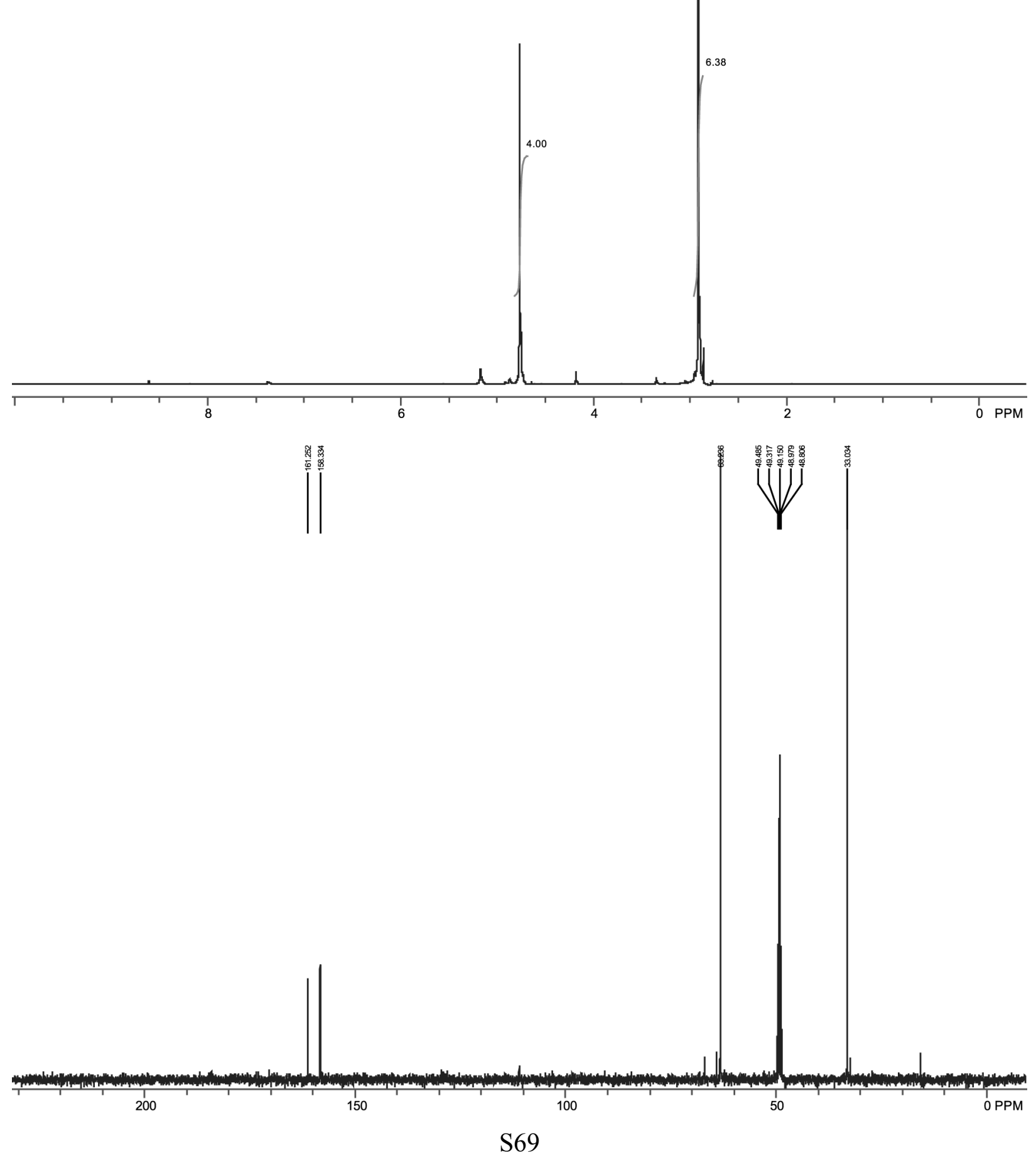
Supporting Information; Appendix 1: Nilsson and Overman 2-(3,5-Dimethyl-4-oxo-[1,3,5]triazinan-1-yl)-6-methyl-4-phenyl-1,4-dihydropyrimidine-5carboxylic acid ethyl ester (Compound 21a).<smiles>CCOC(=O)C1=C(C)NC(N2CN(C)C(=O)N(C)C2)=NC1c1ccccc1</smiles>
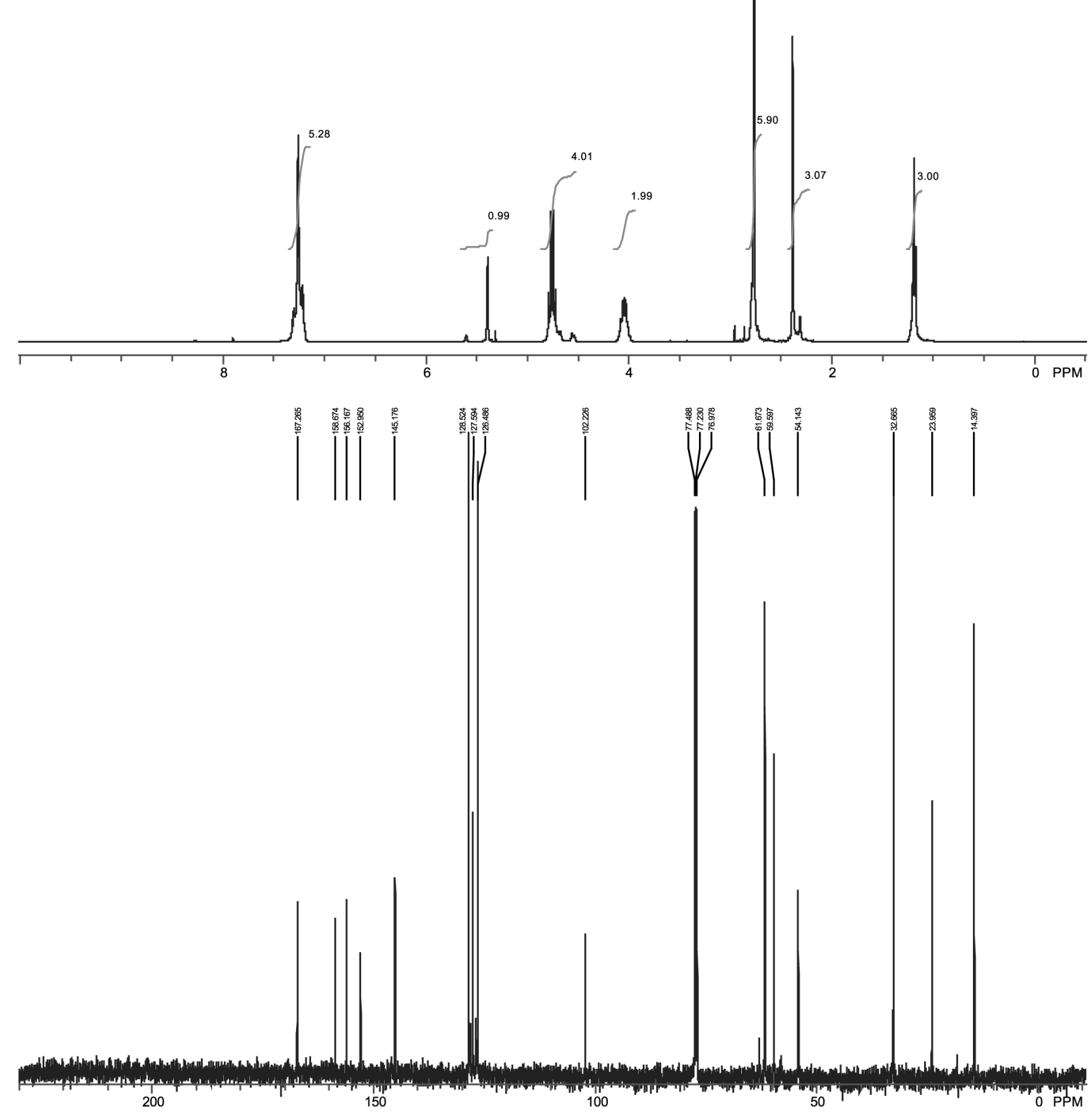
Supporting Information; Appendix 1: Nilsson and Overman 2-(3,5-Dimethyl-4-oxo-[1,3,5]triazinan-1-yl)-6-methyl-4-(2-vinyl-phenyl)-1,4-dihydropyrimidine-5carboxylic acid ethyl ester (Compound 21b).<smiles>C=Cc1ccccc1C1N=C(N2CN(C)C(=O)N(C)C2)NC(C)=C1C(=O)OCC</smiles>
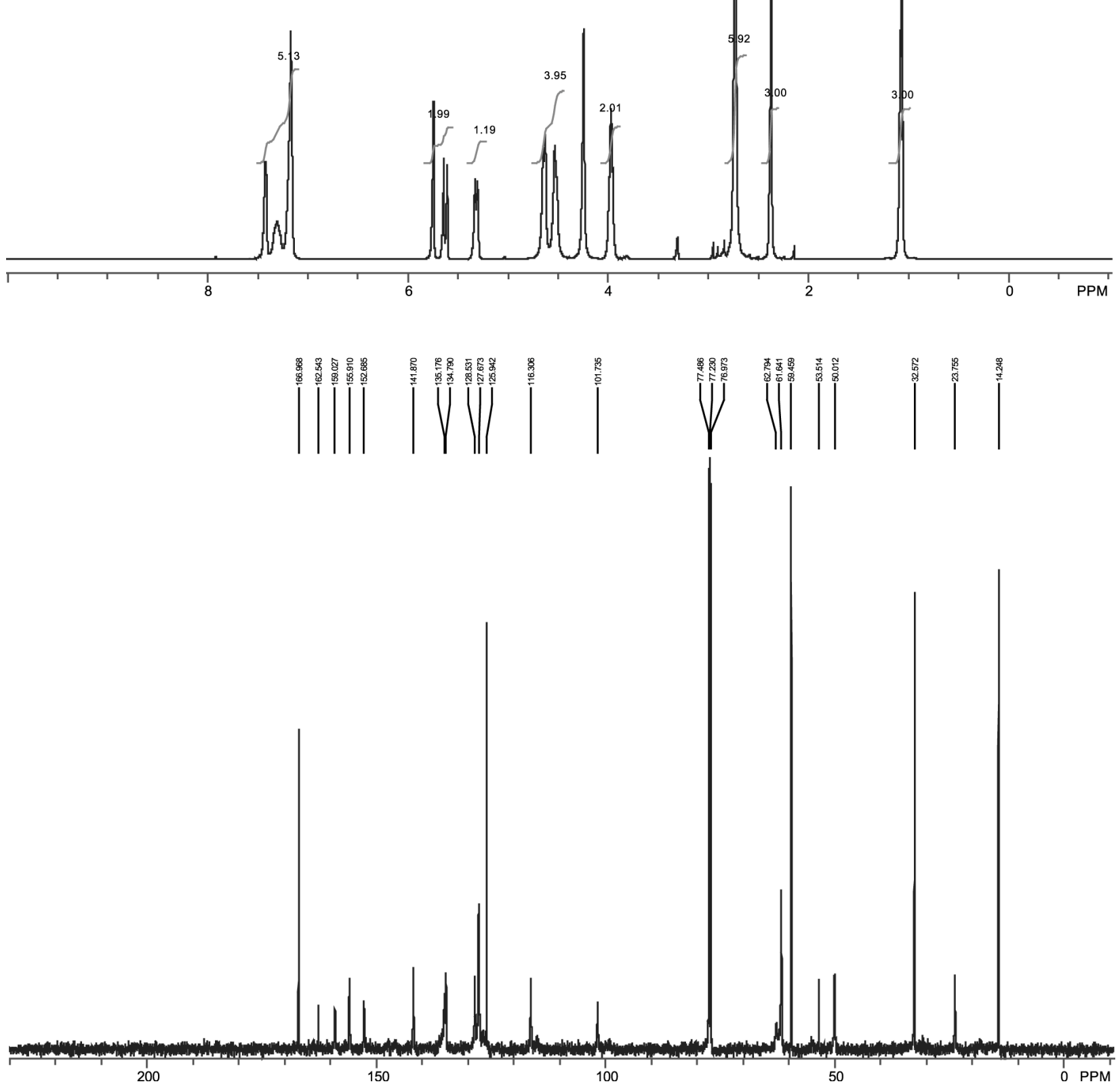
Supporting Information; Appendix 1: Nilsson and Overman 2-(3,5-Dimethyl-4-oxo-[1,3,5]triazinan-1-yl)-4-isopropyl-6-methyl-1,4-dihydropyrimidine-5carboxylic acid benzyl ester (Compound 21c).<smiles>CC1=C(C(=O)OCc2ccccc2)C(C(C)C)N=C(N2CN(C)C(=O)N(C)C2)N1</smiles>
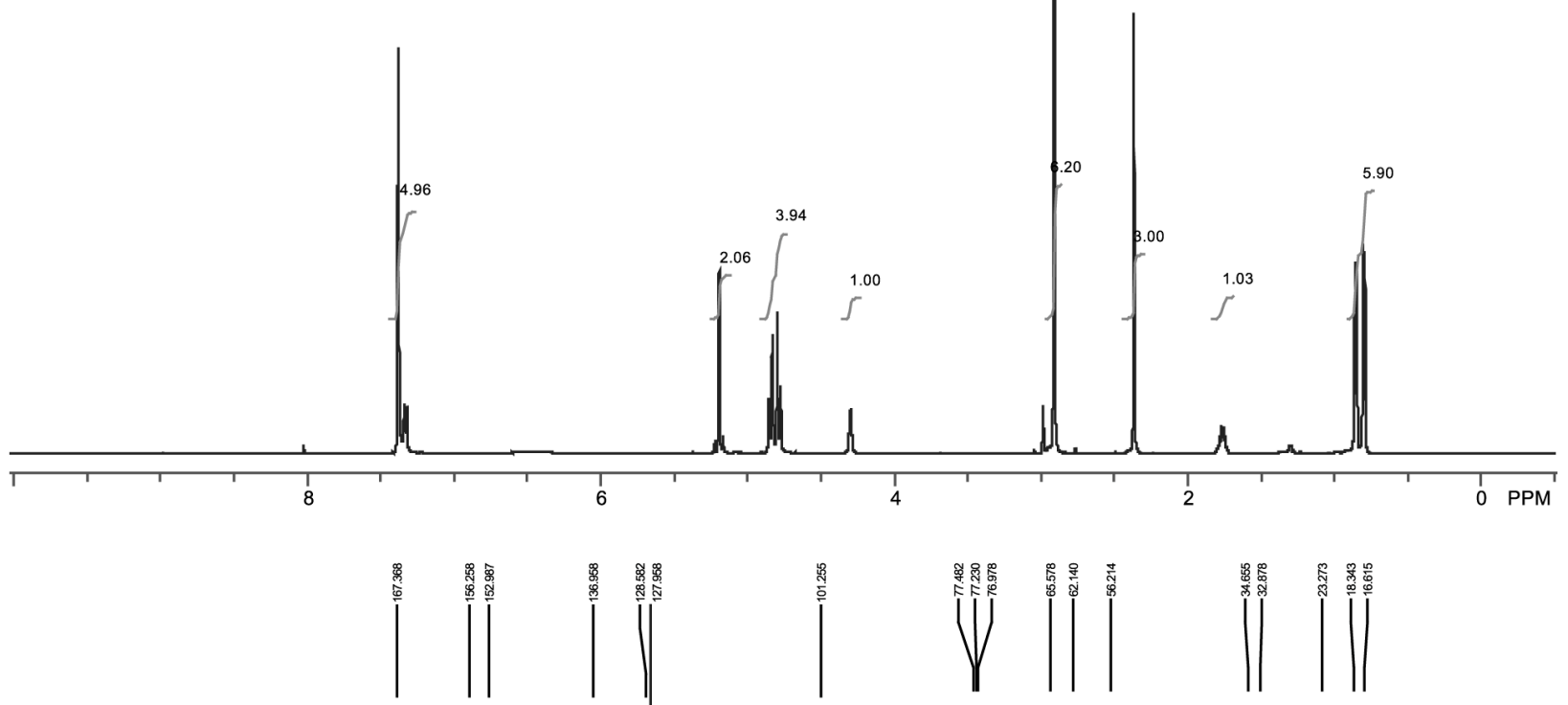
Supporting Information; Appendix 1: Nilsson and Overman 2-(3,5-Dimethyl-4-oxo-[1,3,5]triazinan-1-yl)-6-methyl-4-propyl-1,4-dihydropyrimidine-5carboxylic acid benzyl ester (Compound 21d).

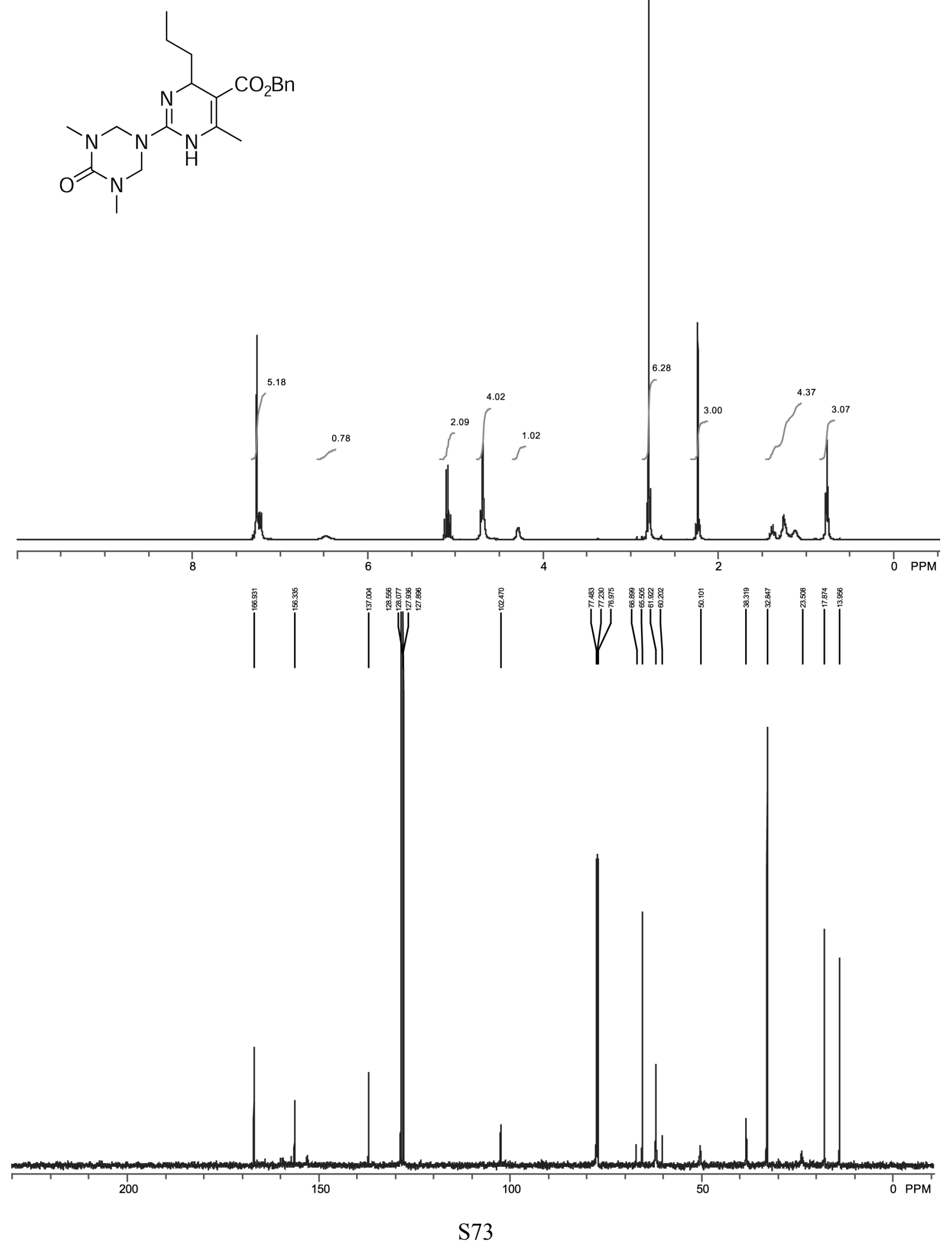


Supporting Information; Appendix 1: Nilsson and Overman 4-Cyclohexyl-2-(3,5-dimethyl-4-oxo-[1,3,5]triazinan-1-yl)-6-methyl-1,4-dihydropyrimidine-5carboxylic acid benzyl ester (Compound 21e).<smiles>CC1=C(C(=O)Cc2ccccc2)C(C2CCCCC2)N=C(N2CN(C)C(=O)N(C)C2)N1</smiles>

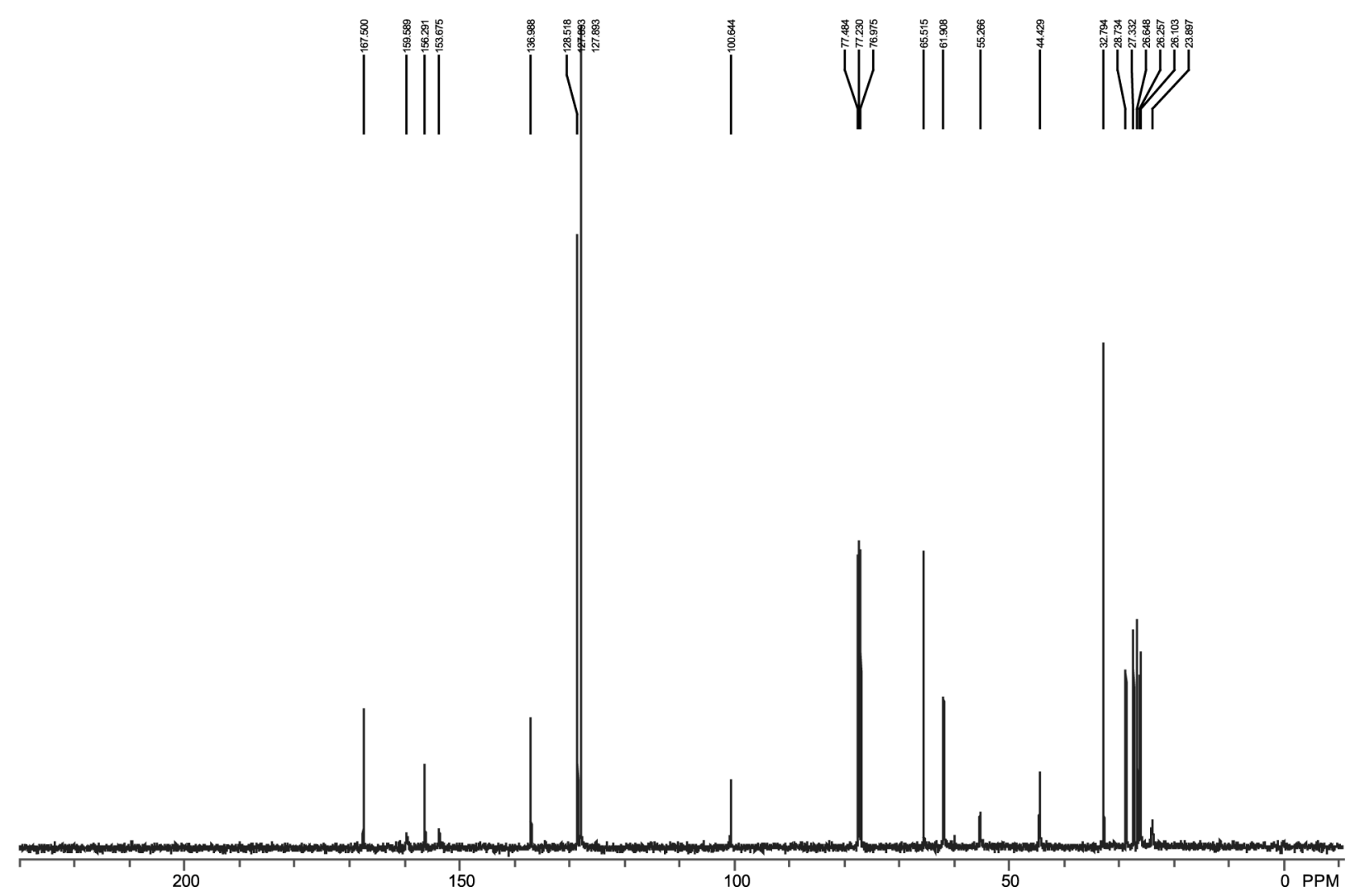


Supporting Information; Appendix 1: Nilsson and Overman 2-(3,5-Dimethyl-4-oxo-[1,3,5]triazinan-1-yl)-6-methyl-4-(3-nitro-phenyl)-1,4-dihydropyrimidine-5carboxylic acid ethyl ester (Compound 21f).

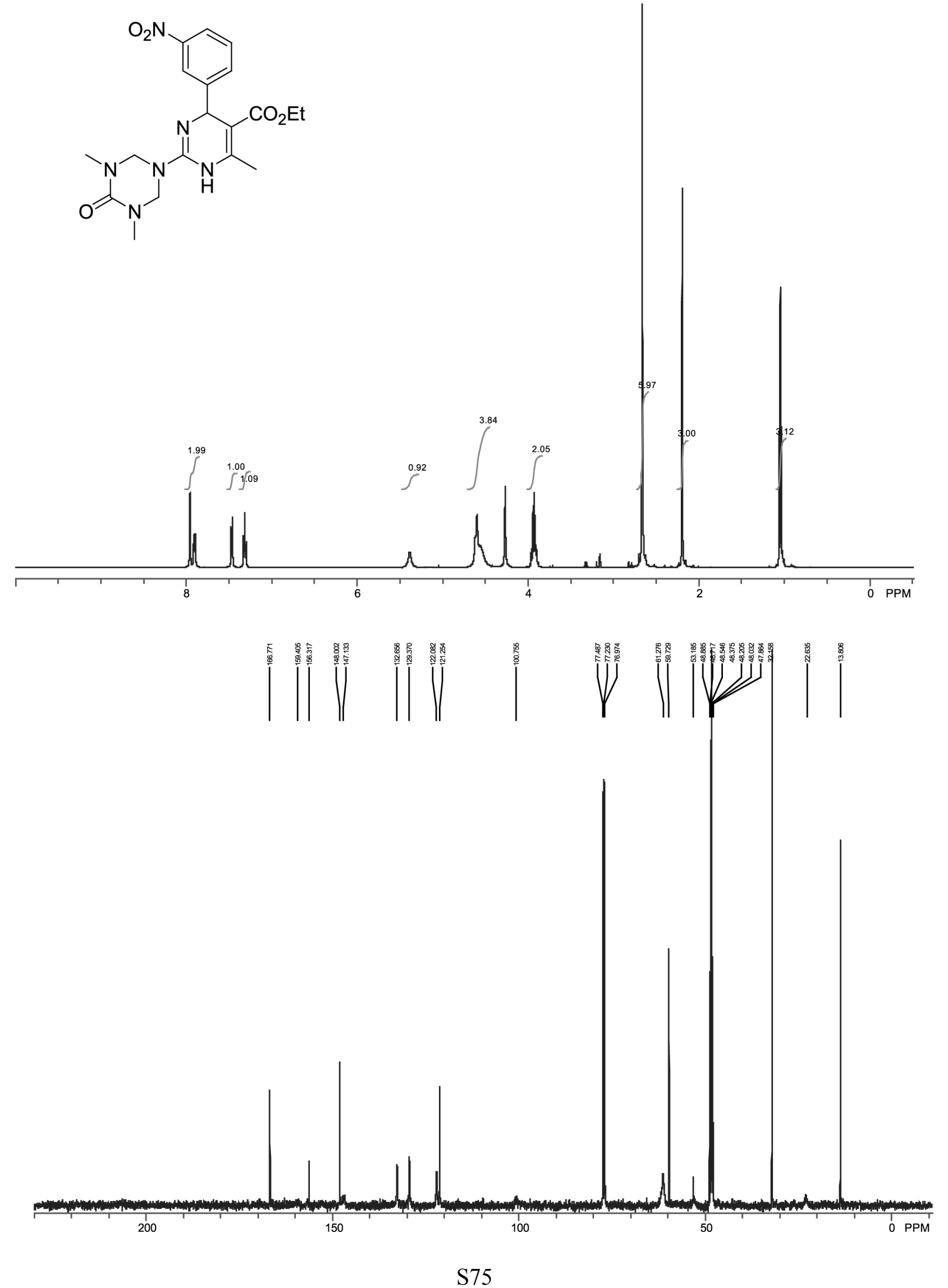


Supporting Information; Appendix 1: Nilsson and Overman 2-(3,5-Dimethyl-4-oxo-[1,3,5]triazinan-1-yl)-4-furan-2-yl-6-methyl-1,4-dihydropyrimidine-5carboxylic acid ethyl ester (Compound 21g).<smiles>CCOC(=O)C1=C(C)NC(N2CN(C)C(=O)N(C)C2)=NC1c1ccco1</smiles>
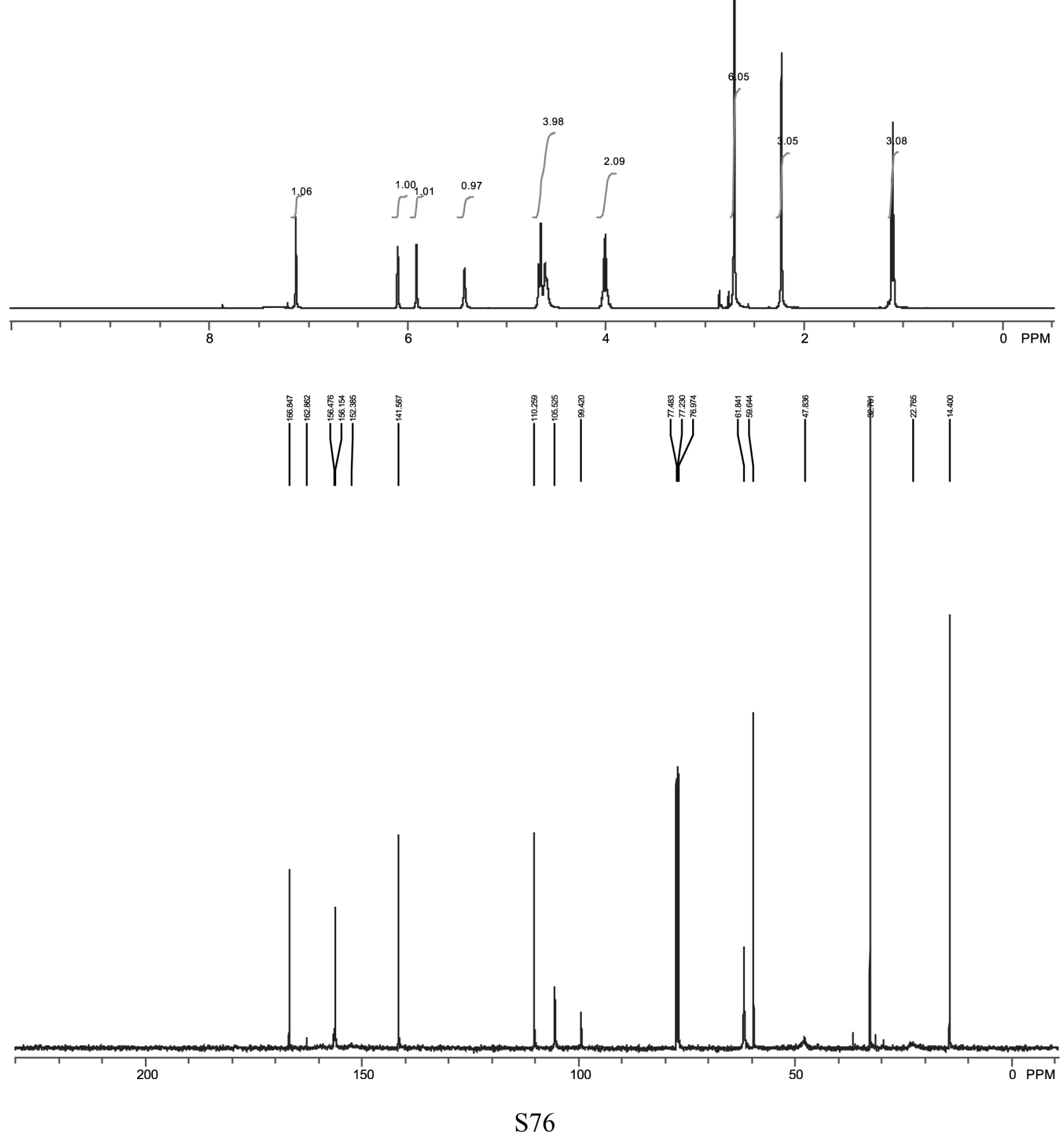
Supporting Information; Appendix 1: Nilsson and Overman 4-tert-Butyl-2-(3,5-dimethyl-4-oxo-[1,3,5]triazinan-1-yl)-6-methyl-1,4-dihydropyrimidine-5carboxylic acid benzyl ester (Compound 21h).<smiles>CC1=C(C(=O)Cc2ccccc2)C(C(C)(C)C)N=C(N2CN(C)C(=O)N(C)C2)N1</smiles>
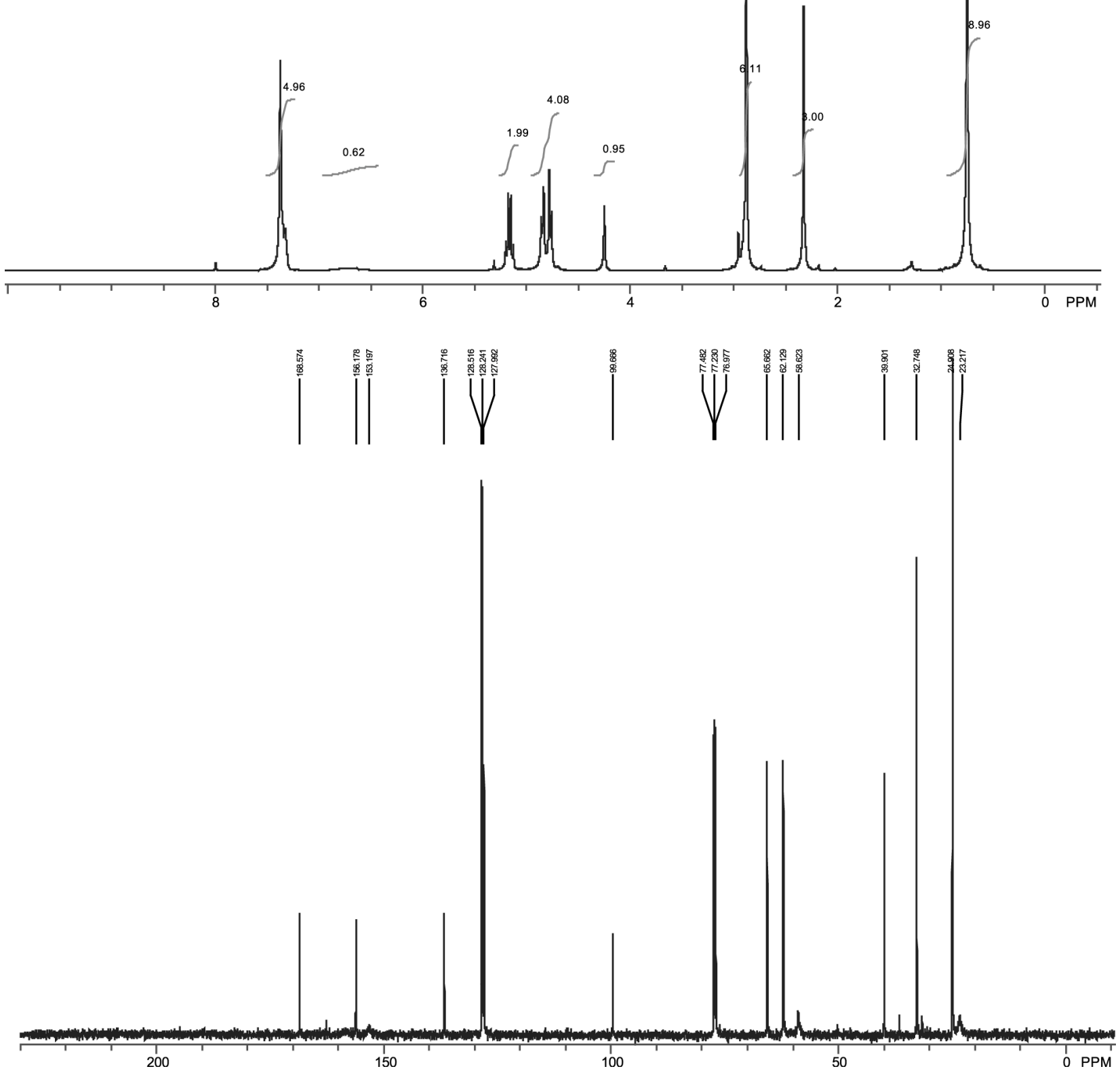
Supporting Information; Appendix 1: Nilsson and Overman 2-(3,5-Dimethyl-4-oxo-[1,3,5]triazinan-1-yl)-6-methyl-4-(4-nitrophenyl)-1,4-dihydropyrimidine-5carboxylic acid ethyl ester (Compound 21i).<smiles>CCOC(=O)C1=C(C)NC(N2CN(C)C(=O)N(C)C2)=NC1c1ccc([N+](=O)[O-])cc1</smiles>
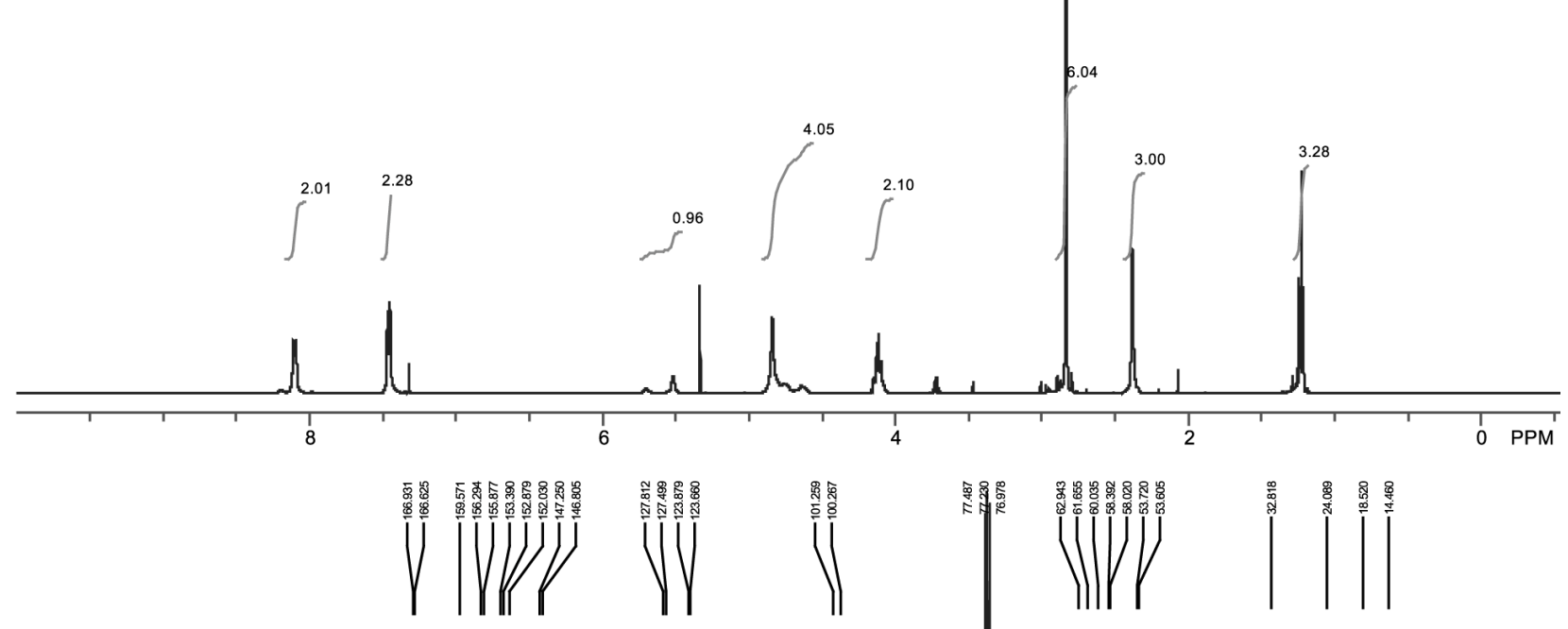

4-(2,4-Dimethoxy-phenyl)-2-(3,5-dimethyl-4-oxo-[1,3,5]triazinan-1-yl)-6-methyl-1,4-dihydropyrimidine-5-carboxylic acid ethyl ester (Compound 21j).<smiles>CCOC(=O)C1=C(C)NC(N2CN(C)C(=O)N(C)C2)=NC1c1ccc(OC)cc1OC</smiles>
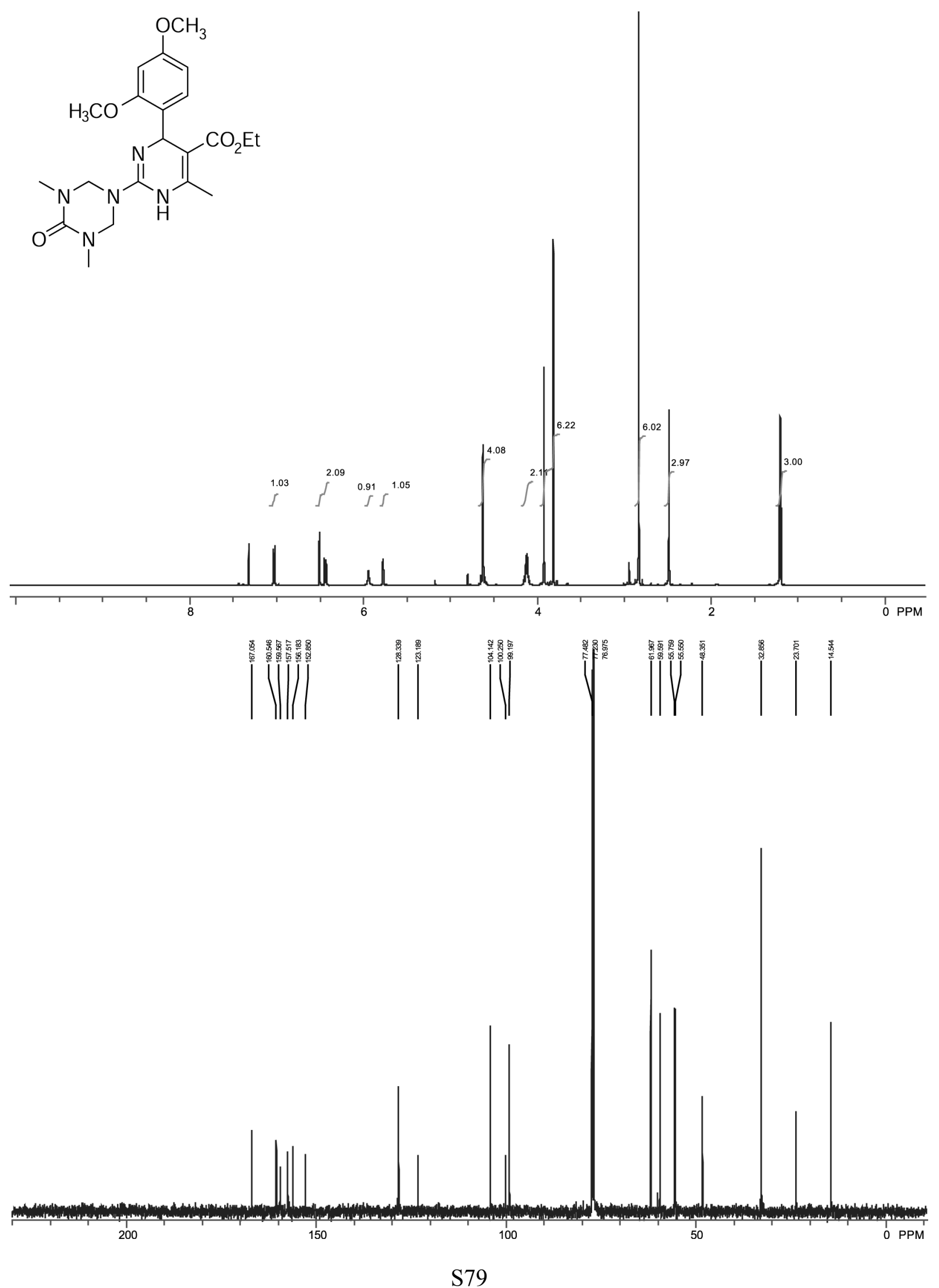